\title{
Flow and Radiation Analyses for Stardust Entry at High Altitude
}

\author{
Iain D. Boyd* \\ University of Michigan, Ann Arbor, Michigan, 48109-2140, USA \\ Jiaqiang Zhong ${ }^{\dagger}$ and Deborah A. Levin ${ }^{\ddagger}$ \\ Pennsylvania State University, University Park, Pennsylvania, 16802, USA \\ Peter Jenniskens ${ }^{\S}$ \\ SETI Institute, Mountain View, California, 94043, USA
}

\begin{abstract}
The Stardust Sample Return Capsule (SRC) entered the Earth's atmosphere at a velocity of $12.6 \mathrm{~km} / \mathrm{s}$. At high altitude, the flow field is in a strong state of thermochemical nonequilibrium. In the present study, both continuum (CFD) and particle (DSMC) methods are used to analyze the flow around the SRC at high altitude. The present study contains two components. In the first part, CFD and DSMC flow fields are generated and used to compute line of sight spectra that are compared with high-resolution data taken with the Echelle spectrograph during the Stardust entry. Comparisons between simulation and measurements are presented for air spectral features and for metal atomic lines believed to originate from evaporation of the vehicle surface. In the second part, detailed DSMC computations are performed at high altitude that include a kinetic model for the excitation processes of sodium.
\end{abstract}

\section{Introduction}

The Stardust payload was launched in February 1999 on a mission to use aerogel to collect samples of material from comet Wild-2. In January 2006, almost exactly seven years later, after collecting the cometary dust and returning to Earth, the Stardust Sample Return Capsule (SRC) entered the atmosphere at a velocity of $12.6 \mathrm{~km} / \mathrm{s}$. This is the highest energy vehicle entry ever undertaken. Shortly after entering the atmosphere, the Stardust SRC landed in Utah allowing its precious scientific payload to be safely recovered.

The forebody thermal protection material used on Stardust was PICA (Phenolic Impregnated Carbon Ablator) that has also been selected as a candidate material for use on NASA's Orion Crew Exploration Vehicle (CEV). The combination of having an entry velocity similar to Mars return, and the use of PICA as heat shield material make it of significant interest to perform detailed analyses of the Stardust SRC entry even after the successful completion of its mission.

A number of studies of the aerothermodynamics of the Stardust SRC were performed prior to the mission. ${ }^{1-4}$ These studies conducted flow field analyses using both continuum CFD methods (by solving the Navier-Stokes equations) and particle methods (using the direct simulation Monte Carlo method, DSMC ${ }^{5}$ ). However, the DSMC study ${ }^{3}$ was focused on the high-altitude aerodynamics of the capsule and did not include the effects of ionization reactions. Recent studies have compared CFD and DSMC simulation results for Stardust at high altitude where ionization was included. ${ }^{6,7}$ This article studies associated radiation phenomena and consists of two parts: the first one conducted primarily at the University of Michigan, and the second primarily at the Pennsylvania State University.

*Professor, Department of Aerospace Engineering, 1320 Beal Ave, AIAA Associate Fellow.

${ }^{\dagger}$ Postdoctoral Fellow, Department of Aerospace Engineering, AIAA Member.

¥Professor, Department of Aerospace Engineering, AIAA Associate Fellow.

$\S$ Research Scientist, Carl Sagan Center, AIAA Senior Member. 


\section{Part I: Modeling of Stardust Radiation}

The primary goal of this part of the study is to perform high-altitude analyses of the radiation environment around the Stardust SRC. Flow field computations are performed with both continuum and particle methods. Direct comparisons between the solutions provided by these techniques allows evaluation of differences between these approaches for modeling the strongly nonequilibrium thermochemical phenomena generated under highly energetic entry conditions.

The geometry of the Stardust SRC, the entry conditions chosen for study, and the spectral data are first described. The two numerical methods employed in the study for flow field computation are described. These consist of a CFD code for solving the Navier-Stokes equations with coupled nonequilibrium thermochemistry, and a DSMC code that also includes thermochemical nonequilibrium. The radiation associated with the flow field is computed using the NEQAIR code. ${ }^{10}$ The results are discussed and final conclusions formulated.

\section{Flow Conditions}

The geometry of the Stardust SRC is shown schematically in Fig. 1. The forebody consists of a 60-deg. half-angle, spherically blunted cone with a nose radius of $0.2202 \mathrm{~m}$. In the computations, a small portion of the shoulder is included that has a radius of $0.02 \mathrm{~m}$. The flow and surface conditions for the two altitudes considered are listed in Table 1. The Knudsen numbers are in the near-continuum regime where only small differences would be expected between CFD and DSMC simulation results.

This study compares computed spectra with data from the Echelle instrument, ${ }^{9}$ a miniature Echelle spectrograph that provided a high spectral resolution over a wide wavelength range from 360 to $880 \mathrm{~nm}$, increasing from FWHM $=0.3$ to $0.9 \mathrm{~nm}$ over this range, and that obtained 100 spectra over the altitude range of 82 to $69 \mathrm{~km}$. The spectra contain a number of interesting features including: (1) an underlying blackbody signal from the heated surface of the Stardust SRC; (2) atomic lines of air-plasma species; (3) atomic lines of metals assumed to originate from the white paint applied to the exterior of the TPS; and (4) band structures from air molecules and cyanide.

\section{Flowfield Simulation Methods}

The two different numerical approaches applied in this study are: (1) CFD solution of the continuum Navier-Stokes equations using the DPLR code; and (2) particle-based DSMC computation. In each case, an 11-species, 19-reaction, thermochemical nonequilibrium approach is adopted. The wall temperature is assumed fixed at a temperature of 2,000 $\mathrm{K}$ for the $81 \mathrm{~km}$ case, and at 2,700 $\mathrm{K}$ for the $71 \mathrm{~km}$ condition. The wall material PICA is assumed to be fully catalytic to ions (that recombine into their neutral atoms and molecules) and atoms (that recombine into molecules). Ablation processes are not included in these studies.

\section{III.A. Details of the Continuum Flow Model}

The continuum computations are performed using the DPLR CFD code. ${ }^{11,12}$ DPLR is a parallel multiblock finite-volume code that solves the Navier-Stokes equations including finite-rate chemistry and the effects of thermal nonequilibrium. The Euler fluxes are computed using a modified (low-dissipation) form of StegerWarming flux vector splitting, ${ }^{13}$ with third-order spatial accuracy obtained via MUSCL extrapolation. ${ }^{14}$ Viscous fluxes are computed to second-order accuracy using a central difference approach. DPLR has been used previously on several other planetary entry simulations. ${ }^{11,15,16}$

The flow is assumed to be in a state of thermochemical nonequilibrium with three separate temperatures for the translational, rotational, and vibrational modes. Viscous transport and thermal conductivity are modeled using the mixing rules presented by Gupta et al. ${ }^{17}$ which have been shown to be reasonable approximations of the more accurate Chapman-Enskog relations in this flow regime. ${ }^{18,19}$ The bifurcation method is used to compute the species diffusion coefficients. ${ }^{20}$ This method allows for the variations in species diffusion coefficients to be accurately modeled without sacrificing the requirement that the diffusion velocities sum identically to zero.

Rotational relaxation is modeled using the relaxation time based on the Parker model. ${ }^{21}$ Vibrational relaxation is modeled using a Landau-Teller formulation, where relaxation times are obtained from Millikan and White, ${ }^{22}$ assuming simple harmonic oscillators, with the high-temperature correction of Park. ${ }^{23}$ The 19-reaction finite-rate air chemistry model uses the rates ${ }^{24,25}$ that are listed in Table 2 . The backward rates 
are computed using the forward rates and equilibrium constants evaluated using the van't Hoff equation. Dissociation reaction rates are computed according to the two-temperature model of Park. ${ }^{23}$

The baseline DPLR case employs the rates in Table 2, the standard Park ${ }^{23}$ two-temperature model for dissociation in which the controlling temperature $T=T_{t}^{0.5} \times T_{v}^{0.5}$, and all other reactions are controlled solely by the translational temperature.

To address differences in chemistry modeling between DPLR and DSMC, use of different controlling temperatures $T=T_{t}^{n} \times T_{v}^{1-n}$ is considered for both dissociation and ionization reactions. For dissociation, heat bath studies indicate that nonequilibrium DSMC dissociation rates are closer to those obtained in DPLR with $n=0.3$ rather than the baseline of $n=0.5$. For direct ionization, in DSMC the collision energy is determined by the relative velocity between the atom and the electron that is generally dominated by the electron velocity. Thus, DSMC simulation of direct ionization is more closely approximated in DPLR using $n=0.0$ assuming that the vibrational temperature in DPLR is a better approximation to the electron temperature than the translational temperature. In presentation of results, the baseline DPLR case is indicated by "b" and the modified chemistry case by " $m$ ".

Surface catalysis is modeled using a diffusion limited approach. ${ }^{23}$ The surface is assumed to be fully catalytic to $\mathrm{N}_{2}$ and $\mathrm{O}_{2}$ recombination, which should be reasonable for the PICA TPS material based on its expected similarity to graphite TPS systems.

\section{III.B. Details of the Particle Flow Model}

The particle computations employ a DSMC code developed specifically for hypersonic, ionized flow simulations. ${ }^{26}$ Models are implemented for rotational ${ }^{27}$ and vibrational ${ }^{28}$ energy exchange that are consistent with those employed in DPLR. Most of the chemical reactions are simulated using the Total Collision Energy (TCE) model except for the dissociation of nitrogen and oxygen. These reactions employ the Vibrationally Favored Dissociation (VFD) model. ${ }^{29}$ The VFD model makes it possible to bias the dissociation reaction probability in favor of molecules that possess a higher vibrational energy. In this way, the important phenomenon of vibration-dissociation coupling can be simulated. In the present work, the VFD favoring parameter $\phi$ is set to 2.0 for nitrogen dissociation and 0.5 for oxygen dissociation, as determined in prior work. ${ }^{29}$ A recent detailed DSMC study of nitrogen dissociation suggests that the VFD model with $\phi=2$ emulates the behavior of a more sophisticated approach. ${ }^{30}$

The forward rate coefficients employed in the DSMC computations are the same as those employed by DPLR that are listed in Table 2. Note that this set of rates is the standard reacting air mechanism employed by DPLR except for the two direct ionization reactions:

$$
\begin{aligned}
& N+E^{-} \rightarrow N^{+}+2 E^{-} \\
& O+E^{-} \rightarrow O^{+}+2 E^{-}
\end{aligned}
$$

It is not possible to use the low, negative temperature exponents of the rates usually employed by DPLR in the DSMC TCE chemistry model. The rates employed here are those reported by Wilson. ${ }^{31}$ The lower activation energies in these rates are based on the premise that most atomic ionization occurs not from the ground electronic state, but rather from the lowest excited states of nitrogen and oxygen.

The backward steps of all chemical reactions are modeled using the approach described by Boyd ${ }^{32}$ that provides consistency with the CFD approach.

The presence of electrons in the flow field presents a challenge for the DSMC technique due to their very small mass and associated very high thermal velocities. Under the assumption of ambi-polar diffusion in which the diffusion rates of electrons and ions are assumed equal, the standard approach in DSMC for handling this situation is to tie each electron particle to the ion particle with which it was created in the ionization process. ${ }^{33}$ In the present highly energetic flow condition, the ionization degree is expected to be significant and keeping track of pairs of electrons and ions can cause difficulties for the standard DSMC approach. Therefore, a model previously developed for DSMC computation of plasma thrusters ${ }^{34}$ is employed here. In this approach, the average ion velocity in each computational cell is evaluated and all electrons in each cell are moved with this average velocity. While the approach does not guarantee charge neutrality, in practice it is found that neutrality is achieved everywhere in the flow domain within a few percent. This approach for handling electrons is significantly more robust than the standard DSMC approach. 


\section{Radiation Analysis}

Line-of-sight radiation is computed from the flow field solutions using the nonequilibrium radiation code, NEQAIR ${ }^{10}$ To populate the excited states, NEQAIR provides options to employ either an equilibrium assumption (Boltzmann) or a finite rate approach under the assumption of a quasi-steady state (QSS). In the present work, the QSS approach is employed for air species, and the Boltzmann model is employed for metal atomic lines. NEQAIR takes as input the temperatures of the translational, rotational, and vibrational modes, the electron temperature, and the number densities of all chemical species. While the DSMC computation simulates these four different temperatures, DPLR does not simulate the electron temperature. In computing spectra based on DPLR solutions, the sensitivity to using either the translational or vibrational temperature to represent the electron temperature is investigated.

\section{Flowfield Results}

\section{V.A. $81 \mathrm{~km}$ Condition}

The meshes used in the DPLR computations consist of three blocks containing $24 \times 128,64 \times 128$, and 48x128 cells. In each case, the mesh is grown out hyperbolically from the vehicle surface to ensure orthogonality and then adapted to contours of Mach number.

The DSMC computations employ a single mesh containing 100 by 200 cells in which the cell dimension in the flow direction is everywhere less than the local mean free path. Typically, about two million particles are employed of which 40,000 are electrons. The timestep used in the DSMC computations is smaller than the mean free time anywhere in the flow field.

Figure 2 provides a comparison of the translational temperature contours obtained with DSMC (upper) and with the baseline DPLR computation (indicated in subsequent plots as "b" while the DPLR case with modified chemistry modeling is indicated as " $\mathrm{m}$ "). Qualitatively, the comparison of temperature contours shows: (1) the shock wave predicted by DSMC is significantly thicker than that simulated by DPLR; and (2) the peak translational temperature from DSMC is significantly higher than that simulated by DPLR.

Quantitative differences between the three simulation cases can be assessed using Fig. 3 that shows temperature profiles along the stagnation streamline. The modifications to the chemistry modeling in DPLR push the results towards better agreement with the DSMC profiles. The changes in the controlling temperatures for dissociation and direct ionization lead to significant increases in the peak temperatures for each of the translational, rotational, and vibrational modes. In addition, the shock thickness is significantly increased. However, there are still significant differences between the DSMC and DPLR-m profiles. Note that DSMC provides the translational temperature of the electrons whereas DPLR assumes that the electrons have the same translational temperature as the bulk gas. The DSMC profile of electron temperature lies between the rotational and vibrational temperature profiles.

Profiles of the number densities of the neutral species are shown in Fig. 4. The DPLR solutions generally show a more compact shock structure and a higher level of dissociation in comparison to the DSMC results. Due to the use of the same fully catalytic surface boundary condition in each simulation, the species concentrations at the wall are in good agreement. Profiles of the number densities of the nitrogen ions and the electrons are shown in Fig. 5. Here there are significant differences between the two DPLR solutions, with the modified case producing significantly less ionization that is in much better agreement with the DSMC profiles. The baseline DPLR solution predicts a peak electron number density that is about a factor of 25-30 higher than the peak predictions from the other two simulations. Note that the baseline DPLR case predicts a very low level of $\mathrm{N}_{2}^{+}$in comparison to the other two simulations.

\section{V.B. $71 \mathrm{~km}$ Condition}

The meshes used in the DPLR computations again consist of three blocks containing $24 \times 128,64 \times 128$, and $48 \times 128$ cells. The DSMC computations employ a single mesh containing 200 by 200 cells in which the cell dimension in the flow direction is everywhere less than the local mean free path. Typically, about two million particles are employed of which 100,000 are electrons. The timestep used in the DSMC computations is smaller than the mean free time anywhere in the flow field.

Quantitative differences between the three simulation cases can be assessed using Fig. 6 that shows temperature profiles along the stagnation streamline. The modifications to the chemistry modeling in DPLR 
again push the results towards better agreement with the DSMC profiles although the sensitivity in the DPLR solutions to chemistry modeling is much reduced.

Profiles of the number densities of the neutral species are shown in Fig. 7. A small distance downstream of the shock, the two DPLR solutions become almost identical. By comparison, DSMC shows lower levels of nitrogen and oxygen dissociation. Profiles of the number densities of the nitrogen ions and the electrons are shown in Fig. 8. The differences between the two DPLR solutions is significantly reduced in comparison to the $81 \mathrm{~km}$ case. For example, the two profiles for the electron number density generally agree well within a factor of two. By comparison, the DSMC profiles show significant differences with either of the DPLR profiles. Significantly, DSMC predicts a peak electron number density that is smaller by about a factor of 10 and a peak number density of $\mathrm{N}_{2}^{+}$that is higher than either of the DPLR results.

\section{Comparisons of Spectra}

\section{VI.A. $81 \mathrm{~km}$ Condition}

In Fig. 9, the full spectrum measured by the Echelle instrument at $81 \mathrm{~km}$ is compared with three simulation results along the stagnation streamline of the flow: (1) DSMC; (2) baseline DPLR with the electron temperature $\left(\mathrm{T}_{e}\right)$ set to the translational temperature $\left(\mathrm{T}_{t}\right)$; and (3) baseline DPLR with the electron temperature set to the vibrational temperature $\left(\mathrm{T}_{v}\right)$. The comparison indicates that there are significant differences between the three computed spectra. As expected, since the translational temperature is always higher than the vibrational temperature, the DPLR-based spectra using $\mathrm{T}_{e}=\mathrm{T}_{t}$ has significantly higher levels of emission.

In Fig. 10, the same comparison is made only here using the two spectra obtained from the modified DPLR simulation. Again there is significant variation between the three simulation results, and by comparison with the DPLR spectra in Fig. 9, it is clear that there is also significant variation among the four DPLR results.

To investigate the spatial variation of the radiation, flow field profiles from all simulations are extracted at several radial locations. The line-of-sight spectra based on each of these profiles are compared. In Fig. 11, comparison is made for four different radial profiles for the baseline DPLR simulation using $\mathrm{T}_{e}=\mathrm{T}_{t}$. There is relatively little variation among these profiles with a maximum difference of less than $20 \%$. The same comparison is made in Fig. 12 with emission evaluated using $\mathrm{T}_{e}=\mathrm{T}_{v}$. The variation in spectra with radius is much more significant in this case, with more than a factor of two difference. The same comparisons are next made for the modified chemistry DPLR simulation in Figs. 13 and 14. Similar levels of differences are found between these cases. Finally, the radial dependence of the DSMC based spectra is shown in Fig. 15. Here, the maximum difference between the spectra is as high as a factor of four. In general, the DPLR based spectra computed using $\mathrm{T}_{e}=\mathrm{T}_{t}$ show a small radial variation, whereas the DPLR spectra using $\mathrm{T}_{e}=\mathrm{T}_{v}$ and the DSMC spectra show considerable variation. The difference between the trends in the DPLR results is explained by the fact that the vibrational temperature generally increases with radius since the flow has more time to undergo vibrational relaxation. At the same time, the region of high vibrational temperature becomes thicker away from the stagnation streamline. Similarly, in the DSMC solution, the peak electron temperature and shock layer thickness both increase with radius due to analogous effects. The trends can be seen in Fig. 16 that shows contours of vibrational temperature from the modified DPLR case (upper) and electron temperature contours from DSMC (lower). By comparison, the translational temperature contours from the DPLR simulations, see Fig. 2, show relatively little variation in magnitude and thickness with radius.

The Echelle instrument of course has no spatial resolution, and so area weighting is employed to derive spatially-averaged, computed spectra that are shown in the remaining comparisons.

To better assess the various spectral predictions, comparisons are next provided over more limited wavelength ranges. In Fig. 17, spectra are shown in the range of 370-420 nm. In the measured spectra, this region contains a strong signal attributed to cyanide, CN, in the range of $385-389 \mathrm{~nm}$, as well as a peak at around $391.4 \mathrm{~nm}$ attributed to a band-head of the $\mathrm{N}_{2}^{+}$molecular system. None of the flow field simulations (DPLR and DSMC) include carbon chemistry and so comparison with the CN emission is not possible. In addition, the measured $\mathrm{CN}$ emission appears to overwhelm some of the $\mathrm{N}_{2}^{+}$emission features. The DPLR spectra shown in Fig. 17 are for the baseline chemistry. In comparison to the Echelle data, the DSMC spectrum over predicts the $\mathrm{N}_{2}^{+}$band-head while the DPLR spectrum using $\mathrm{T}_{e}=\mathrm{T}_{t}$ provides almost perfect agreement. The DPLR spectrum using $\mathrm{T}_{e}=\mathrm{T}_{v}$ significantly under predicts the feature. The DPLR spectra shown in Fig. 18 are from the modified chemistry simulation. In this case, both DPLR spectra over predict the band-head and the DPLR result using $\mathrm{T}_{e}=\mathrm{T}_{v}$ is in good agreement with the DSMC result. The over prediction of the 
$\mathrm{N}_{2}^{+}$emission by these simulations can be traced to the significantly higher levels of $\mathrm{N}_{2}^{+}$indicated in Fig. 5 . These comparisons indicate that there is significant uncertainty in the prediction of $\mathrm{N}_{2}^{+}$chemistry at this flow condition.

In Fig. 19, spectra are shown in the range of $810-880 \mathrm{~nm}$ that contains several lines of atomic nitrogen and oxygen. Careful examination of these atomic spectral features indicates that the baseline DPLR result using $\mathrm{T}_{e}=\mathrm{T}_{t}$ is in good agreement with the DSMC spectrum and that both agree well with the measured Echelle data. By comparison, the baseline DPLR result using $\mathrm{T}_{e}=\mathrm{T}_{v}$ under predicts the atomic line intensities. The corresponding comparison is provided in Fig. 20 for the modified DPLR simulation. In this case, the DPLR result using $\mathrm{T}_{e}=\mathrm{T}_{t}$ significantly over predicts the atomic line intensities while the DPLR solution with $\mathrm{T}_{e}=\mathrm{T}_{v}$ provides good agreement with both DSMC and the Echelle data.

Similar comparisons are made in the wavelength range of 740-780 nm in Figs. 21 and 22. Strong lines of potassium are observed in the Echelle data in this portion of the spectrum. Focusing first on the air atomic lines, the conclusions are again that DSMC agrees quite well with the baseline DPLR solution using $\mathrm{T}_{e}=\mathrm{T}_{t}$, the modified DPLR solution using $\mathrm{T}_{e}=\mathrm{T}_{v}$, and with the Echelle data.

Attention is now turned to the potassium lines shown in Figs. 21 and 22. The Echelle spectra at nearly all altitudes show strong signals from several metallic atoms including potassium, zinc, and sodium. It is believed that some of these metals originate in the paint coating the SRC and some come from the PICA. To study the generation of these emissions, a series of DSMC computations is performed in which a flux of metal atoms is assumed to out-gas at the wall temperature $(2,000 \mathrm{~K})$ from all surfaces of the SRC forebody at a radially uniform rate. Profiles of the resulting flow field are extracted and input into NEQAIR that is run using the Boltzmann option since detailed rates for all excited states are not available in the literature. Through comparison of the resulting spectra with the Echelle data, the out-gas flux is adjusted until good agreement is obtained. Radial variation in these spectra is again observed and the results presented are area averaged across the SRC forebody.

The DSMC results for the potassium lines shown in Figs. 21 and 22 indicate very good agreement with the Echelle data. The uniform surface flux required to obtain this level of agreement is $1.04 \times 10^{20}$ particles $/ \mathrm{m}^{2} / \mathrm{s}$. Numerical studies of PICA ablation performed at NASA Ames Research Center ${ }^{35}$ indicate a total mass flux of material blowing off the surface at this flow condition of $1.26 \times 10^{-2} \mathrm{~kg} / \mathrm{m}^{2} / \mathrm{s}$. Thus, the above potassium flux corresponds to a blowing mass fraction of about $530 \times 10^{-6}$. Analysis of PICA using a mass spectrometer, also conducted at NASA Ames Research Center, ${ }^{36}$ indicates that the mass fraction of potassium in PICA is in the range of $25-50 \times 10^{-6}$. Hence, the enhanced rate of potassium flux from the surface is likely not from PICA alone. It is reasonable to associate the additional flux with the evaporation of the Z-93P paint applied on top of the PICA. It should be noted that this paint contains potassium silicate.

A similar study is conducted for zinc and the results are shown in Fig. 23. The measured line intensity is reproduced well using the DSMC flow field with a uniform surface flux of $9.29 \times 10^{20}$ particles $/ \mathrm{m}^{2} / \mathrm{s}$, that is much higher than the potassium out-gassing rate. This flux of zinc corresponds to a blowing mass fraction of about $8,270 \times 10^{-6}$. The mass spectrometer analysis of PICA indicates that the mass fraction of zinc is about $3 \times 10^{-6}$. Clearly, the high level of zinc emission observed in the Echelle spectra does not originate in the PICA material, and presumably again comes from the paint that includes zinc oxide pigment. It is noted in passing that the Echelle spectrum includes strong emission from a triplet of zinc lines centered around $472 \mathrm{~nm}$. Unfortunately, NEQAIR cannot predict intensities for these lines since no transition probabilities could be found in the literature.

The third metal considered is sodium for which the final results are shown in Fig. 24. The required surface flux for sodium is $1.43 \times 10^{19}$ particles $/ \mathrm{m}^{2} / \mathrm{s}$ that corresponds to a blowing mass fraction of $40 \times$ $10^{-6}$. The mass spectrometer analysis of PICA reports sodium mass fractions ranging from about 40 to 300 $\times 10^{-6}$. In this case, the required surface flux of sodium lies close enough to the measured range that it is reasonable to conclude that the PICA TPS is the most likely source of this metal impurity.

The Boltzmann assumption assumed for computation of emissions from the metal atoms is known to overpredict air emissions. However, the peak emission from the metal impurities occurs immediately adjacent to the surface due to the steep density gradient of these species. Since this is the location where the flow is closest to being in equilibrium, due to the relatively high total number density there, it is likely that the Boltzmann approach for the metal species is a better approximation than it is for the air species. 


\section{VI.B. $\quad 71 \mathrm{~km}$ Condition}

In Fig. 25, the full spectrum measured by the Echelle instrument at $71 \mathrm{~km}$ is compared with three simulation results along the stagnation streamline of the flow: (1) DSMC; $(2)$ modified DPLR with the electron temperature $\left(\mathrm{T}_{e}\right)$ set to the translational temperature $\left(\mathrm{T}_{t}\right)$; and (3) modified DPLR with the electron temperature set to the vibrational temperature $\left(\mathrm{T}_{v}\right)$. The comparison indicates a significant reduction in the sensitivity of the DPLR-based spectra to the electron temperature. In addition, the DPLR-based spectra using the baseline DPLR chemistry model gives almost identical profiles to those shown here. Thus, for the remainder of this section, only DPLR-based spectra using the modified chemistry modeling are shown. There are clearly still significant differences between the DSMC and DPLR-based spectra in Fig. 25.

Radial variation of the spectra computed at $71 \mathrm{~km}$ are very similar to those discussed earlier at $81 \mathrm{~km}$. The remaining spectra presented below are again spatially averaged across the face of the capsule.

In Fig. 26, spectra are shown in the range of 370-420 nm. In comparison to the Echelle data, the DSMC spectrum again over predicts the $\mathrm{N}_{2}^{+}$band-head while the DPLR spectrum using $\mathrm{T}_{e}=\mathrm{T}_{t}$ provides good agreement. The DPLR spectrum using $\mathrm{T}_{e}=\mathrm{T}_{v}$ significantly under predicts the feature.

In Fig. 27, spectra are shown in the range of 810-880 $\mathrm{nm}$ that contains several lines of atomic nitrogen and oxygen. The comparisons between predicted and measured spectra are not always consistent for the various features. In most cases, the DPLR result obtained with $\mathrm{T}_{e}=\mathrm{T}_{t}$ is in good agreement with the the measured spectra and the result obtained with $\mathrm{T}_{e}=\mathrm{T}_{v}$ is a little lower. In general, the DSMC based results lie below the measured emission.

Similar comparisons are made in the wavelength range of 740-780 nm in Fig. 28. Again, focusing first on the air atomic lines, the conclusions are similar to those stated above for Fig. 27. The Echelle spectrum again shows two clear potassium lines in Fig. 28. The predicted total mass flux of ablated PICA material at this altitude is $9.86 \times 10^{-3} \mathrm{~kg} / \mathrm{m}^{2} / \mathrm{s}^{35}$ Using the same blowing mass fraction of potassium that was employed to obtain good agreement with the Echelle potassium data at $81 \mathrm{~km}$, the DSMC result in Fig. 28 again matches the peak emission measured at the lower wavelength. The predicted peak for the higher wavelength feature is under predicted in comparison to the measured data.

Using the same blowing mass fraction employed at $81 \mathrm{~km}$ for zinc produces the results shown in Fig. 29. The Echelle data do not show any signal from zinc at $71 \mathrm{~km}$ and this is qualitatively consistent with the DSMC result. Finally, the spectra for sodium are shown in Fig. 30. Similar to zinc, employing the same mass fraction here as was used at $81 \mathrm{~km}$ leads to a predicted signal from sodium that lies below the background signal in the Echelle data. Once again, all that can be said is that the DSMC prediction is qualitatively consistent with the lack of the sodium feature in the measured spectrum.

\section{Summary}

The high altitude portion of the Stardust entry trajectory generated very high energy, strongly nonequilibrium flow conditions. In the present study, both continuum (DPLR) and particle (DSMC) simulation methods were employed to generate flow field solutions for Stardust at an altitude of $81 \mathrm{~km}$. Two sets of DPLR results were generated: (1) a baseline case using the default chemistry model; and (2) a modified case in which the temperatures used to determine the dissociation and ionization rates were altered to be more consistent with the DSMC chemistry modeling approach. Significant differences in the temperatures and number densities were found in the two DPLR solutions with the modified case providing better agreement with the DSMC results. For example, the baseline DPLR chemistry model predicted a peak electron number density that was a factor of 25 higher than the peaks predicted by the modified DPLR chemistry model and by DSMC. It is concluded that significant uncertainty exists in the chemistry modeling of such highly energetic, nonequilbrium flows.

The DPLR and DSMC flow field results were used as input to the nonequilibrium radiation code, NEQAIR, to compute emission spectra. NEQAIR requires as input all the species number densities, and the translational, rotational, vibrational, and electron temperatures. The DPLR computations do not explicitly simulate an electron temperature and so spectra were computed by assuming that the electron temperature is given by either the translational temperature or the vibrational temperature. In all cases, the emissions computed using the translational temperature were significantly higher than those obtained using the vibrational temperature.

At this flight condition, lines of sight extracted along different radial coordinates produced varying levels of emission. While the spectra computed using DPLR and the translational temperature displayed modest 
variations with radial location, the DPLR spectra based on vibrational temperature and the DSMC spectra, that are based on electron temperature, showed variations by factors of $2-4$. These variations are mainly explained by the fact that both the vibrational temperature and electron temperature tend to increase with radial distance from the axis. In addition, the axial extent of high levels of vibrational and electron temperature increase with radius. Each of these effects is created by the nonequilbrium phenomena in this relatively low-density flow condition.

The radially varying spectra were weighted by area in order to make quantitative comparisons with the spectra measured during the Stardust entry by the Echelle instrument. For atomic air lines, three different flow field simulations gave good agreement with the measured features: (1) DSMC; (2) baseline DPLR using the translational temperature; and (3) modified DPLR using the vibrational temperature. The fact that similar spectra were obtained from three different flow fields that show significant differences is both encouraging and disturbing. Further work is needed to determine the generality of this result and to identify the explanation.

An interesting aspect of the Echelle measurements involves very strong emission signals from several different metallic lines. Additional DSMC computations were performed in which fluxes from the Stardust surface of potassium, zinc, and sodium were included. By extracting lines of sight from the resulting flow fields, and employing the Boltzmann emission feature of NEQAIR, spectra were obtained for these metals. The magnitudes of the metal fluxes from the surface were varied until good agreement was found between the computed and measured line intensities. These studies indicated that the surface fluxes for potassium and zinc that were required to generate the emissions measured by Echelle are much higher than can be accounted for by impurities in the PICA thermal protection material that covered the Stardust capsule. It is therefore concluded that these emissions arise from the white paint that covered the PICA which is known to contain both potassium silicate and zinc oxide. For sodium, the flux inferred from the simulations was on the same order of magnitude as the impurity concentration for PICA and so this signal is concluded to arise from ablation of the TPS material.

Similar analysis conducted at $71 \mathrm{~km}$ showed significantly less sensitivity to chemistry modeling in the DPLR results. In general, the DPLR-based spectra provided better comparison to the air-species features present in the measured spectra than did the DSMC-based spectra. In terms of the metal impurities, use of the same mass fractions for blowing from the surface that were established in the $81 \mathrm{~km}$ analysis led to good agreement with the measured potassium signal at $71 \mathrm{~km}$. For zinc and sodium, no clear features were measured at $71 \mathrm{~km}$. This is in qualitative agreement with the simulation results that predicted the signals from these metals to lie below the measured background intensity.

\section{Acknowledgments}

The work of IDB was sponsored in part by the Stanford Center for Turbulence Research, and the Eloret Corporation through NASA Contract NNA04BC25C. IDB gratefully acknowledges the assistance of Kerry Trumble and Dinesh Prabhu, both of the Eloret Corporation, in obtaining the DPLR and NEQAIR results, respectively.

\section{Part II: Modeling of Stardust Sodium Ablation Flows}

\section{Introduction}

In our recent work we have studied the high-altitude, transitional to continuum reentry flow of Stardust in terms of developing new excitation models appropriate to $\mathrm{DSMC}^{6}$ as well as modeling the importance of thermal and chemical ablation. ${ }^{38}$ In the first work we studied the change of the flowfield between the altitudes of 100 to $70 \mathrm{~km}$ and showed that ultra-high Mach number reentry vehicles create sufficiently energetic flow conditions with substantial degrees of ionization of 1 to $11 \%$ occurring in the non-continuum flow regime. To model these transitional, ionized, and thermochemically nonequilibrium flows, a DSMC approach was investigated and energy exchange models that have been developed for Navier-Stokes computational fluid dynamics computations were examined and revised for application to the DSMC method. Building on this work, in Ref. 38 the importance of the ablation process of the Stardust thermal protection layer on the flow field and surface parameters was investigated using the direct simulation Monte Carlo method to model surface thermal and chemical ablation processes as well as ionization processes. It was found that chemical 
ablation due to the reaction between solid carbon and gaseous oxygen and nitrogen atoms is dominant over thermal ablation for the early portion of the reentry. As the altitude decreases, the forebody surface temperature increases, the ablation process becomes more intensive, and the influence of ionization reactions becomes more important due to a denser free stream condition.

In this work, we utilize the models that were developed in these two earlier papers to study a spectroscopic feature observed in the Stardust reentry flow Echelle spectral data of Jennenkins, ${ }^{9}$ shown in Fig. 24. The figure shows the radiation from the sodium doublet of ${ }^{2} \mathrm{P}_{3 / 2,1 / 2}$ which is due to the outgassing or thermal ablation of sodium from the Stardust TPS PICA material. Laboratory reports provided by Stackpoole ${ }^{36}$ showed that for two unflown samples of PICA studied in ICP ground based testing, 168 to 308 ppm of sodium have been observed. The next largest atomic species outgassed was seen to be Scandium. Sodium radiation has been observed from numerous hypersonic flights and is virtually impossible to quench. ${ }^{39}$ Since sodium does not exist in the Mesospheric portion of the atmosphere the modeling of this radiation may only be due to outgassing from the TPS material. Comparison of the predicted radiation with that observed, although spatially averaged over the entire vehicle, provides confirmation of the modeling of the hypersonic boundary layer and its interaction with a charring ablator thermal protection material. To simulate sodium ablation from Stardust, a kinetic or quasi-steady state (QSS) model must be proposed because there are not sufficient collisions to populate the sodium electronic states in a Boltzmann distribution. The QSS model includes the populations of the sodium ground state as well as the first two electronically excited states of ${ }^{2} \mathrm{P}_{1 / 2}$ and ${ }^{2} \mathrm{D}_{5 / 2,3 / 2}$. As shown in previous work, ${ }^{40}$ an overlay approach wherein the electronically excited state species populations are computed based on a converged gas dynamic solution will be employed. This approach should be valid in the free-molecular to near-continuum flight regime because the fraction of population in the excited states are sufficiently low as to not effect the flowfield solution.

\section{Kinetic Model for Excited Sodium State Populations}

Extensive experimental studies of atomic, excited and ionized sodium cross sections exist for many of the important excitation and ionization processes and are discussed, for example, in Zecca et al. ${ }^{41}$ Ground state atomic sodium can be excited to the higher energy levels of ${ }^{2} \mathrm{P}_{1 / 2}\left(16964 . \mathrm{cm}^{-1}\right)$ and ${ }^{2} \mathrm{D}_{5 / 2}(29172.8$ $\left.\mathrm{cm}^{-1}\right) .{ }^{42}$ The excited states of ${ }^{2} \mathrm{P}_{1 / 2}$ and ${ }^{2} \mathrm{D}_{5 / 2}$ have a lifetime of about 16.4 and $20.0 \mathrm{nsec}$ before the excited electron radiates its energy and transitions back down to the ground state. The following collisionally induced excitation and ionization processes are considered because they potentially impact the distributions of sodium ground and excitation states, ${ }^{43}$

$$
\begin{gathered}
\mathrm{O}_{2}+\mathrm{Na}(\mathrm{i}) \rightarrow \mathrm{O}_{2}+\mathrm{Na}^{+}(\mathrm{i})+\mathrm{e}^{-} \\
\mathrm{N}_{2}+\mathrm{Na}(\mathrm{i}) \rightarrow \mathrm{N}_{2}+\mathrm{Na}^{+}(\mathrm{i})+\mathrm{e}^{-} \\
\mathrm{O}+\mathrm{Na}(\mathrm{i}) \rightarrow \mathrm{O}+\mathrm{Na}^{+}(\mathrm{i})+\mathrm{e}^{-} \\
\mathrm{N}+\mathrm{Na}(\mathrm{i}) \rightarrow \mathrm{N}+\mathrm{Na}^{+}(\mathrm{i})+\mathrm{e}^{-} \\
\mathrm{e}^{-}+\mathrm{Na}(\mathrm{g}) \rightarrow \mathrm{e}^{-}+\mathrm{Na}(1) \\
\mathrm{e}^{-}+\mathrm{Na}(1) \rightarrow \mathrm{e}^{-}+\mathrm{Na}(2) \\
\mathrm{e}^{-}+\mathrm{Na}(\mathrm{i}) \rightarrow \mathrm{e}^{-}+\mathrm{Na}^{+}(\mathrm{i})+\mathrm{e}^{-} \\
\mathrm{M}+\mathrm{Na}(1) \rightarrow \mathrm{M}+\mathrm{Na}(\mathrm{g}) \\
\mathrm{M}+\mathrm{Na}(2) \rightarrow \mathrm{M}+\mathrm{Na}(1) \\
\mathrm{Na}(1) \rightarrow \mathrm{Na}(\mathrm{g})+\mathrm{h} \nu_{1} \\
\mathrm{Na}(2) \rightarrow \mathrm{Na}(1)+\mathrm{h} \nu_{2}
\end{gathered}
$$

The symbol $M$ represents all neutral species (except sodium), and the symbol $i$ can have a value of $g, 1$, and 2 representing sodium ground or excited states of ${ }^{2} \mathrm{P}_{1 / 2}$ and ${ }^{2} \mathrm{D}_{5 / 2}$, respectively. The rate coefficient values and expressions that will be used in the QSS model are given in Table 3. Note that the translational and electron temperatures derived from the DSMC calculations are used in evaluating the rate coefficients given in Eqs. (1)-(4) and Eqs. (5)-(7), respectively. 
Based on the simple hard sphere model, the forward reaction rate as a function of reaction collision cross section can be expressed as, ${ }^{5}$

$$
k_{f}=\frac{\sigma_{R}}{\epsilon}\left(\frac{8 k T}{\pi m_{r}}\right)^{0.5} \exp \left(-\frac{E_{a}}{k T}\right)
$$

where $\epsilon$ is a symmetric factor that is equal to unity for reactions (5) - (9). The last two radiation reactions (10) and (11) have a reaction rate of

$$
k_{f}=\frac{h \nu}{\tau}
$$

where $\tau$ is the lifetime of the excited state.

Using Eqs. (1) -(13) above and Table 3, the time-dependent change of sodium excited state ${ }^{2} \mathrm{P}_{1 / 2}$ and ${ }^{2} \mathrm{D}_{5 / 2}$ atomic concentration may be expressed as,

$$
\begin{aligned}
& \frac{d[\mathrm{Na}(1)]}{d t}=k_{f, 5}\left[e^{-}\right][\mathrm{Na}(g)]-A_{2}[\mathrm{Na}(1)] \\
& \frac{d[\mathrm{Na}(2)]}{d t}=k_{f, 6}\left[e^{-}\right][\mathrm{Na}(1)]-A_{3}[\mathrm{Na}(2)]
\end{aligned}
$$

where

$$
\begin{aligned}
& A_{2}=k_{f, 1}\left[\mathrm{O}_{2}\right]+k_{f, 2}\left[\mathrm{~N}_{2}\right]+k_{f, 3}[\mathrm{O}]+k_{f, 4}[\mathrm{~N}]+k_{f, 7}\left[\mathrm{e}^{-}\right]+k_{f, 8}[M]+k_{f, 10}\left[\mathrm{e}^{-}\right] \\
& A_{3}=k_{f, 1}\left[\mathrm{O}_{2}\right]+k_{f, 2}\left[\mathrm{~N}_{2}\right]+k_{f, 3}[\mathrm{O}]+k_{f, 4}[\mathrm{~N}]+k_{f, 7}\left[\mathrm{e}^{-}\right]+k_{f, 9}[M]+k_{f, 11}\left[\mathrm{e}^{-}\right]
\end{aligned}
$$

Based on the conservation principle,

$$
[\mathrm{Na}(\mathrm{g})]+[\mathrm{Na}(1)]+[\mathrm{Na}(2)]=[\mathrm{Na}] .
$$

Using the total sodium number density, $[\mathrm{Na}]$, from the DSMC solution, the sodium number densities of the ground and excited states can be obtained from solving linear Eqs. (16)-(18) for the non-equilibrium state. The radiation intensity as a function of the excited sodium number density, $N_{u}$, can be calculated as,

$$
I_{\lambda}=\frac{N_{u} h c}{\tau \lambda} .
$$

As mentioned above, if there were sufficient collisions such that the system was in a fully equilibrium state, the number of atom at ground and excited states would satisfy a Boltzmann distribution,

$$
N_{i}=\frac{g_{i} e^{-E_{i} / k T}}{Q}
$$

where

$$
Q=\sum_{i=0}^{n} g_{i} e^{-E_{i} / k T}
$$

and the subscript $i$ refers to the ground or excited state, $g_{i}$ is the degeneracy of electronic state $i$, and $E_{i}$ is the energy level of that state (or the required excitation energy to reach the $i$-th state).

\section{DSMC and QSS Simulation Results}

The DSMC based SMILE software ${ }^{49}$ is used in this work to model the 2-D axisymmetric Stardust sodium ablation calculations at $81 \mathrm{~km}$. The freestream and Stardust surface conditions as well as DSMC computational parameters are summarized in Table 4. Cell adaption occurs automatically with up to 100 subcell division enabled so that the flow mean free path is approximately on the same order of local cell size. The Stardust forebody stagnation point is located at the origin of the coordinate system. The air species chemical and ionization reactions were summarized in Ref. 37 Note that any potential reactions of the ablated sodium with the air-derived flowfield species are neglected in the DSMC modeling. The assumption is that although such collisions may affect the sodium excited state population, they are too few to effect the gas dynamics. ${ }^{40}$ 
In order to test the implementation technique of modeling sodium ablation from a hypersonic, blunt body surface, such as Stardust, numerical comparisons were performed between two DSMC codes, SMILE and Monaco. Figure 31 shows a comparison between sodium number density, velocity and temperature along the forebody stagnation streamline with a surface ablation rate of $3.5 \times 10^{19}$ per $\mathrm{m}^{2}$ per second into a vacuum environment for the two DSMC codes. In addition, in SMILE, two numerical techniques, full-spherical sources and half-spherical sources, both placed in cells adjacent to the wall, were used and tested to model sodium ablation from surface. It can be seen that either of these two SMILE techniques produce results that agree well with those of Monaco. With adequate grid resolution, either SMILE approach will work because the full spherical source particles move towards and collide essentially immediately with the wall. Since the wall is assumed to be fully thermally accommodated and diffuse, the reflected particles are expected to behave exactly the same as the half-spherical source molecules moving towards the upstream. The DSMC calculations discussed below were calculated with the SMILE code.

The DSMC solutions of total, sodium and electron number densities along the forebody (top) and afterbody (bottom) stagnation line at $81 \mathrm{~km}$ are shown in Fig. 32. Using the species number densities from the DSMC simulation, the sodium number densities in the ground and excited states can be obtained by solving the linear set of Eqs. (16)-(18) at the steady state condition. The flowfield distributions of sodium atoms in the ground and excited ${ }^{2} \mathrm{P}_{3 / 2,1 / 2}$ and ${ }^{2} \mathrm{D}_{5 / 2}$ states are shown in Fig. 33, and the solutions along the forebody and afterbody stagnation lines are shown in Fig. 34. It can be seen that the number density of the ground state sodium atom is about 5 to 6 orders of magnitude greater than that of the ${ }^{2} \mathrm{P}_{1 / 2}$ excited state, and the ${ }^{2} \mathrm{P}_{1 / 2}$ excited state sodium atom is about 4 to 6 orders of magnitude greater than the ${ }^{2} \mathrm{D}_{5 / 2}$ excited state. The simulation results suggest that the sodium number density in the excited ${ }^{2} \mathrm{P}_{1 / 2}$ state is in the range of $10^{9}$ to $10^{12}$ atoms per $\mathrm{m}^{3}$, for the surface blowing rate assumed in this work. Finally, the importance of using a QSS model to calculate the populations of the electronically excited states is shown in Fig. 35. It may be seen that both in the bow shock and the wake flows, an assumption of Boltzmann distribution would over-predict the upper state populations by orders of magnitude.

\section{References}

\footnotetext{
${ }^{1}$ Olynick, D., Chen, Y.-K., and Tauber, M. E., "Aerothermodynamics of the Stardust Sample Return Capsule," Journal of Spacecraft and Rockets, Vol. 36, 1999, pp. 442-462.

${ }^{2}$ Mitcheltree, R. A., Wilmoth, R. G., Cheatwood, F. M., Brauckmann, G. J., and Greene, F. A., "Aerodynamics of Stardust Sample Return Capsule," Journal of Spacecraft and Rockets, Vol. 36, 1999, pp. 429-435.

${ }^{3}$ Wilmoth, R. G., Mitcheltree, R. A., and Moss, J. N., "Low-Density Aerodynamics of the Stardust Sample Return Capsule," Journal of Spacecraft and Rockets, Vol. 36, 1999, pp. 436-441.

${ }^{4}$ Gupta, R. N., "Aerothermodynamic Analysis of Stardust Sample Return Capsule With Coupled Radiation and Ablation," Journal of Spacecraft and Rockets, Vol. 37, 2000, pp. 507-514.

${ }^{5}$ Bird, G. A., Molecular Gas Dynamics and the Direct Simulation of Gas Flows Oxford University Press, Oxford, 1994.

${ }^{6}$ Ozawa, T., Zhong, J., Levin, D. A., Boger, D., and Wright, M. J., "Modeling of the Stardust Reentry Flows With Ionization in DSMC," AIAA Paper 2007-0611, January 2007, submitted for publication.

${ }^{7}$ Boyd, I. D., Trumble, K., and Wright, M.J., "Nonequilibrium Particle and Continuum Analyses of Stardust Entry for Near-Continuum Conditions," AIAA Paper 2007-4543, June 2007.

${ }^{8}$ Lofthouse, A. J., Scalabrin, L. C., and Boyd, I. D., "Continuum Breakdown, Velocity Slip and Temperature Jump in Hypersonic Aerothermodynamics," AIAA Paper 2007-0208, January 2007.

${ }^{9}$ Jenniskens, P., "Observations of the STARDUST Sample Return Capsule Entry with a Slit-Less Echelle Spectrograph," AIAA Paper 2008-1210, January 2008.

${ }^{10}$ Whiting, E. E., Park, C., Liu, Y., Arnold, J. O., and Paterson, J. A., "NEQAIR96, Nonequilibrium and Equilibrium Radiative Transport and Spectra Program: User's Manual," NASA Reference Publication 1389, December 1996.

${ }^{11}$ Wright, M., Loomis, M., and Papadopoulos, P., "Aerothermal Analysis of the Project Fire II Afterbody Flow," Journal of Thermophysics and Heat Transfer, Vol. 17, No. 2, 2003, pp. 240-249.

${ }^{12}$ Wright, M., Candler, G., and Bose, D., "Data-Parallel Line Relaxation Method for the Navier-Stokes Equations," AIAA Journal, Vol. 36, No. 9, 1998, pp. 1603-1609.

${ }^{13}$ MacCormack, R. and Candler, G., "The Solution of the Navier-Stokes Equations Using Gauss-Seidel Line Relaxation," Computers and Fluids, Vol. 17, No. 1, 1989, pp. 135-150.

${ }^{14}$ Yee, H., "A Class of High-Resolution Explicit and Implicit Shock Capturing Methods," NASA TM 101088, Feb. 1989.

${ }^{15}$ Wercinski, P., Chen, Y.-K., Loomis, M., Tauber, M., McDaniel, R., Wright, M., Papadopoulos, P., Allen, G., and Yang, L., "Neptune Aerocapture Entry Vehicle Preliminary Design," AIAA Paper No. 2002-4812, Aug. 2002.

${ }^{16}$ Wright, M., Bose, D., and Olejniczak, J., "The Impact of Flowfield Radiation Coupling on Aeroheating for Titan Aerocapture," Journal of Thermophysics and Heat Transfer, Vol. 19, No. 1, 2005, pp. 17-27.

${ }^{17}$ Gupta, R., Yos, J., Thompson, R., and Lee, K., "A Review of Reaction Rates and Thermodynamic and Transport
} 
Properties for an 11-Species Air Model for Chemical and Thermal Nonequilibrium Calculations to 30000 K," NASA RP-1232, Aug. 1990.

${ }^{18}$ Palmer, G. E. and Wright, M. J., "A Comparison of Methods to Compute High Temperature Gas Viscosity," Journal of Thermophysics and Heat Transfer, Vol. 17, No. 2, 2003, pp. 232-239.

${ }^{19}$ Palmer, G. E. and Wright, M. J., "A Comparison of Methods to Compute High Temperature Gas Thermal Conductivity," AIAA Paper No. 2003-3913, Jun. 2003.

${ }^{20}$ Bartlett, E. P., Kendal, R. M., and Rindal, R. A., "An Analysis of the Coupled Chemically Reacting Boundary Layer and Charring Ablator: Part IV - A Unified Approximation for Mixture Transport Properties for Multicomponent Boundary-Layer Applications," NASA CR-1063, June 1968.

${ }^{21}$ Parker, J. G., "Rotational and Vibrational Relaxation in Diatomic Gases," Physics of Fluids, Vol. 2, 1959, pp. 449-462.

${ }^{22}$ Millikan, R. and White, D., "Systematics of Vibrational Relaxation," Journal of Chemical Physics, Vol. 39, No. 12, 1963, pp. 3209-3213.

${ }^{23}$ Park, C., Nonequilibrium Hypersonic Aerothermodynamics, Wiley, New York, 1990.

${ }^{24}$ Park, C., "Review of Chemical-Kinetic Problems of Future NASA Missions, I: Earth Entries", Journal of Thermophysics and Heat Transfer, Vol. 7, No. 3, 1993, pp. 385-398.

${ }^{25}$ Bose D. and Candler, G., "Thermal Rate Constants of the N2 + O =ED NO + N Reaction Using Ab Initio 3A" and 3A' Potential Energy Surfaces," Journal of Chemical Physics, Vol. 104, No. 8, 1996, pp. 2825-2833.

${ }^{26}$ Boyd, I. D. and Gokcen, T., "Computation of Axisymmetric and Ionized Hypersonic Flows Using Particle and Continuum Method," AIAA Journal, Vol. 32, 1994, pp. 1828-1837.

${ }^{27}$ Boyd, I. D., "Analysis of Rotational Nonequilibrium in Standing Shock Waves of Nitrogen," AIAA Journal Vol. 28, 1990, pp. 1997-1999.

${ }^{28}$ Boyd, I. D., "Analysis of Vibrational-Translational Energy Transfer Using the Direct Simulation Monte Carlo Method," Physics of Fluids A, Vol. 3, 1991, pp. 1785-1791.

${ }^{29}$ Haas, B. L. and Boyd, I. D., "Models for Direct Monte Carlo Simulation of Coupled Vibration-Dissociation," Physics of Fluids A, Vol. 5, 1993, pp. 478-489.

${ }^{30}$ Bondar, Y. and Ivanov, M., "DSMC Dissociation Model Based on Two-Temperature Chemical Rate Constant," AIAA Paper 2007-0614, January 2007.

${ }^{31}$ Wilson, J., "Ionization Rate of Air Behind High-Speed Shock Waves," Physics of Fluids, Vol. 9, 1966, pp. $1913-1921$.

${ }^{32}$ Boyd, I. D., "Modeling Backward Chemical Rate Processes in the Direct Simulation Monte Carlo Method," Physics of Fluids, Vol. 19, 2007, article 126103.

${ }^{33}$ Bird, G. A., "Nonequilibrium Radiation During Re-entry at 10 km/s," AIAA Paper 87-1543, June 1987.

${ }^{34}$ Boyd, I. D., "Monte Carlo Simulation of Nonequilibrium Flow in Low Power Hydrogen Arcjets," Physics of Fluids, Vol. 9, 1997, pp. 3086-3095.

${ }^{35}$ Cozmuta, I., private communication, October 2007.

${ }^{36}$ Stackpoole, M., Laboratory reports of ICP/Mass spectra for PICA TPS material samples, private communication.

${ }^{37}$ Takashi Ozawa, Jiaqiang Zhong, Deborah Levin, David Boger, and Michael Wright, "Modeling of Stardust Reentry Flows with Ionization in DSMC," AIAA paper 2007-0611, 2007, submitted for publication.

${ }^{38}$ Jiaqiang Zhong, Takashi Ozawa, and Deborah A. Levin, "Modeling of Stardust Reentry Reacting Thermal and Chemical Ablation Flow," AIAA paper 2007-4551, 2007, submitted for publication.

${ }^{39}$ G. A. Harvey, "Photometric Analysis of Spectrograms of Two Trailblazer I Payload Reentry Events", NASA Technical Note, NASA TN D-3389, Apr. 1966.

${ }^{40}$ D. Levin, G. Candler, and R. Collins, "An Overlay Method for Calculating Excited State Species Properties in Hypersonic Flows," Journal of AIAA, Vol. 35, No.2, p. 288 February 1997.

${ }^{41}$ A. Zecca, G. P. Karwasz, and R. S. Brusa, "One century of experiments on electron-atom and molecule scattering: a critical review of integral cross sections," Rivista Del Nuovo Cimento, Vol. 19, No. 2, pp. 1-146, 1996.

${ }^{42}$ S. G. Rautian, and A. S. Yatsenko, "Grotrian Diagrams," Physics - Uspekhi, Vol. 42, No. 2, pp. 205-208, 1999.

${ }^{43}$ I. S. Zaslonko, and S. M. Kogarko, "Excitation of Sodium in Certain Reactions Behind Shock Waves," Combustion, Explosion and Shock Waves, Vol. 6, No. 2, pp. 175-182, 1970.

${ }^{44}$ M. Lenard, "Ionization of Cesium and Sodium Contaminated Air in the Hypersonic Slender Body Boundary Layer," Space Sciences Laboratory, Missile and Space Division, No. R64SD22, Aug. 1964.

${ }^{45}$ P. M. Stone, and Y. Kim, "Electron-Impact Cross Sections for Ground State to np Excitations of Sodium and Potassium," Journal of Research of the National Institute of Standards and Technology, Vol. 109, No. 5, pp. 505-515, 2004.

${ }^{46}$ K. Fujii, and S. K. Srivastava, "A Measurement of the Electron-impact Ionization Cross Section of Sodium," J. Phys. B: At. Mol. Opt. Phys., Vol. 28, pp. L595-L563, 1995.

${ }^{47}$ W. S. Tan, Z. Shi, C. H. Yang, and L. Vuskovic, "Electron-impact Ionization of Laser-excited Sodium Atom," J. Physical Review A, Vol. 54, No. 5, pp. 3710-3713, Nov. 1996.

${ }^{48}$ I. V. Hertel H. Hofmann, and K. A. Rost, "Electronic to Vibrational-Rotational Energy Transfer in Collisions of Na with SIMPLE Molecules," Chemical Physics Letters, Vol. 47, No. 1, pp. 163-167, Apr. 1977.

${ }^{49}$ M. S. Ivanov, G. N. Markelov, and S. F. Gimelshein, "Statistical simulation of reactive rarefied flows: numerical approach and application", AIAA Paper 98-2669, June 1998 
Table 1. Freestream and surface parameters.

\begin{tabular}{ccc}
\hline \hline & \multicolumn{2}{c}{ Altitude, $\mathrm{km}$} \\
Parameter & 81 & 71 \\
\hline Freestream Temperature, K & 217.6 & 221.6 \\
Freestream Number density, molecules $/ \mathrm{m}^{3}$ & $2.643 \times 10^{20}$ & $1.156 \times 10^{21}$ \\
Freestream Velocity, m/sec & 12385 & 12063 \\
Freestream $\mathrm{O}_{2}$ mole fraction, $\%$ & 23.67 & 23.67 \\
Freestream $\mathrm{N}_{2}$ mole fraction, $\%$ & 76.23 & 76.23 \\
Freestream Knudsen Number, & 0.005 & 0.001 \\
Surface Temperature, $\mathrm{K}$ & 2000 & 2700 \\
& & \\
\hline
\end{tabular}

Table 2. Forward reaction rate coefficients $\left(\mathrm{m}^{3} / \mathrm{molecule} / \mathrm{s}\right)$ used in CFD and DSMC computations.

\begin{tabular}{ccc} 
Number & Reaction & Rate Coefficient \\
\hline $1 \mathrm{M}$ & $\mathrm{N}_{2}+\mathrm{M} \Leftrightarrow \mathrm{N}+\mathrm{N}+\mathrm{M}$ & $1.162 \times 10^{-8} \mathrm{~T}^{-1.6} \exp (-113,200 / \mathrm{T})$ \\
$1 \mathrm{~A}$ & $\mathrm{~N}_{2}+\mathrm{A} \Leftrightarrow \mathrm{N}+\mathrm{N}+\mathrm{A}$ & $4.980 \times 10^{-8} \mathrm{~T}^{-1.6} \exp (-113,200 / \mathrm{T})$ \\
$1 \mathrm{E}$ & $\mathrm{N}_{2}+\mathrm{E}^{-} \Leftrightarrow \mathrm{N}+\mathrm{N}+\mathrm{A}$ & $4.980 \times 10^{-6} \mathrm{~T}^{-1.6} \exp (-113,200 / \mathrm{T})$ \\
$2 \mathrm{M}$ & $\mathrm{O}_{2}+\mathrm{M} \Leftrightarrow \mathrm{O}+\mathrm{O}+\mathrm{M}$ & $3.321 \times 10^{-9} \mathrm{~T}^{-1.5} \exp (-59,400 / \mathrm{T})$ \\
$2 \mathrm{~A}$ & $\mathrm{O}_{2}+\mathrm{A} \Leftrightarrow \mathrm{O}+\mathrm{O}+\mathrm{A}$ & $1.660 \times 10^{-8} \mathrm{~T}^{-1.5} \exp (-59,400 / \mathrm{T})$ \\
$3 \mathrm{M}$ & $\mathrm{NO}+\mathrm{M} \Leftrightarrow \mathrm{N}+\mathrm{O}+\mathrm{M}$ & $8.302 \times 10^{-15} \exp (-75,500 / \mathrm{T})$ \\
$3 \mathrm{~A}$ & $\mathrm{NO}+\mathrm{A} \Leftrightarrow \mathrm{N}+\mathrm{O}+\mathrm{A}$ & $1.826 \times 10^{-13} \exp (-75,500 / \mathrm{T})$ \\
4 & $\mathrm{O}+\mathrm{NO} \Leftrightarrow \mathrm{N}+\mathrm{O}_{2}$ & $1.389 \times 10^{-17} \exp (-19,700 / \mathrm{T})$ \\
5 & $\mathrm{O}+\mathrm{N}_{2} \Leftrightarrow \mathrm{N}+\mathrm{NO}$ & $1.069 \times 10^{-12} \mathrm{~T}^{-1.000} \exp (-37,500 / \mathrm{T})$ \\
6 & $\mathrm{~N}+\mathrm{N} \Leftrightarrow \mathrm{N}_{2}^{+}+\mathrm{E}^{-}$ & $3.387 \times 10^{-17} \exp (-67,700 / \mathrm{T})$ \\
7 & $\mathrm{O}+\mathrm{O} \Leftrightarrow \mathrm{O}_{2}^{+}+\mathrm{E}^{-}$ & $1.859 \times 10^{-17} \exp (-81,200 / \mathrm{T})$ \\
8 & $\mathrm{~N}+\mathrm{O} \Leftrightarrow \mathrm{NO}^{+}+\mathrm{E}^{-}$ & $8.766 \times 10^{-18} \exp (-32,000 / \mathrm{T})$ \\
9 & $\mathrm{~N}+\mathrm{E}^{-} \rightarrow \mathrm{N}^{+}+2 \mathrm{E}^{-}$ & $8.434 \times 10^{-14} \exp (-121,000 / \mathrm{T})$ \\
10 & $\mathrm{O}+\mathrm{E}^{-} \rightarrow \mathrm{O}^{+}+2 \mathrm{E}^{-}$ & $1.054 \times 10^{-14} \exp (-106,200 / \mathrm{T})$ \\
11 & $\mathrm{~N}_{2}+\mathrm{O}^{+} \Leftrightarrow \mathrm{O}+\mathrm{N}_{2}^{+}$ & $1.511 \times 10^{-18} \mathrm{~T}^{0.360} \exp (-22,800 / \mathrm{T})$ \\
12 & $\mathrm{NO}+\mathrm{O}^{+} \Leftrightarrow \mathrm{O}_{2}+\mathrm{N}^{+}$ & $2.324 \times 10^{-25} \mathrm{~T}^{1.900} \exp (-15,300 / \mathrm{T})$ \\
13 & $\mathrm{O}_{2}+\mathrm{NO}^{+} \Leftrightarrow \mathrm{NO}^{+} \mathrm{O}_{2}^{+}$ & $3.985 \times 10^{-17} \mathrm{~T}^{0.410} \exp (-32,600 / \mathrm{T})$ \\
14 & $\mathrm{~N}+\mathrm{NO}^{+} \Leftrightarrow \mathrm{O}+\mathrm{N}_{2}^{+}$ & $1.195 \times 10^{-16} \exp (-35,500 / \mathrm{T})$ \\
15 & $\mathrm{O}+\mathrm{NO}^{+} \Leftrightarrow \mathrm{O}_{2}+\mathrm{N}^{+}$ & $1.660 \times 10^{-18} \mathrm{~T}^{0.500} \exp (-77,200 / \mathrm{T})$ \\
16 & $\mathrm{~N}+\mathrm{O}_{2}^{+} \Leftrightarrow \mathrm{O}_{2}+\mathrm{N}^{+}$ & $1.444 \times 10^{-16} \mathrm{~T}^{0.140} \exp (-28,600 / \mathrm{T})$ \\
17 & $\mathrm{~N}_{2}+\mathrm{O}_{2}^{+} \Leftrightarrow \mathrm{O}_{2}+\mathrm{N}_{2}^{+}$ & $1.644 \times 10^{-17} \exp (-40,700 / \mathrm{T})$ \\
18 & $\mathrm{~N}+\mathrm{NO}^{+} \Leftrightarrow \mathrm{N}_{2}+\mathrm{O}^{+}$ & $5.645 \times 10^{-17} \mathrm{~T}^{-1.080} \exp (-12,800 / \mathrm{T})$ \\
19 & $\mathrm{O}+\mathrm{NO}^{+} \Leftrightarrow \mathrm{N}+\mathrm{O}_{2}^{+}$ & $1.195 \times 10^{-17} \mathrm{~T}^{0.290} \exp (-48,600 / \mathrm{T})$ \\
\hline & &
\end{tabular}


Table 3. Processes and expressions for rate coefficients and cross sections used in QSS model for sodium ablation Excitation Process Expression Reference

\begin{tabular}{lll}
\hline $\begin{array}{l}\text { ionization rate coefficient: } \\
\text { (1) and (2) }\end{array}$ & $k_{f}=6.88 \times 10^{-14} \exp \left(\frac{6.88 \times 10^{-19}}{k T}\right)$ & Ref. 44 \\
$\begin{array}{l}\text { ionization rate coefficient: } \\
(3) \text { and (4) }\end{array}$ & $k_{f}=7.65 \times 10^{-14} \exp \left(\frac{6.88 \times 10^{-19}}{k T}\right)$. & Ref. 44 \\
$\begin{array}{l}\text { electron impact excitation } \\
\text { cross section: (5) and (6) }\end{array}$ & $\sigma_{r}=1.0 \times 10^{-20} \mathrm{~m}^{2}$ & Ref. 45 \\
$\begin{array}{l}\text { electron impact ionization } \\
\text { cross section: (7) }\end{array}$ & $\sigma_{r}=1.0 \times 10^{-22} \mathrm{~m}^{2}$ & Refs. 46,47 \\
quenching cross sections: & $\sigma_{r}=3.0 \times 10^{-19} \mathrm{~m}^{2}$ & Ref. 48 \\
$(8)$ and (9) & & \\
\hline \hline
\end{tabular}

Table 4. Freestream and surface parameters at $81 \mathrm{~km}$.

\begin{tabular}{cc}
\hline \hline & Altitude, $\mathrm{km}$ \\
Parameter & 81 \\
\hline Freestream Temperature, $\mathrm{K}$ & 217.6 \\
Freestream Number density, molecules $/ \mathrm{m}^{3}$ & $2.6388 \times 10^{20}$ \\
Freestream Velocity, $\mathrm{m} / \mathrm{sec}$ & 12391.8 \\
Freestream $\mathrm{O}_{2}$ mole fraction, $\%$ & 23.67 \\
Freestream $\mathrm{N}_{2}$ mole fraction, $\%$ & 76.23 \\
Surface Temperature, $\mathrm{K}$ & 2000 \\
Surface Sodium Ablation Rate, molecules $/\left(\mathrm{m}^{2} / \mathrm{sec}\right)$ & $1.43 \times 10^{19}$ \\
DSMC Computational Domain & \\
axial direction, $\mathrm{m}$ & $-0.15-3.05$ \\
Radial direction, $\mathrm{m}$ & $0 .-0.8$ \\
cell size, $\mathrm{m}$ & $1.5 \times 10^{-3}$ \\
& \\
\hline
\end{tabular}




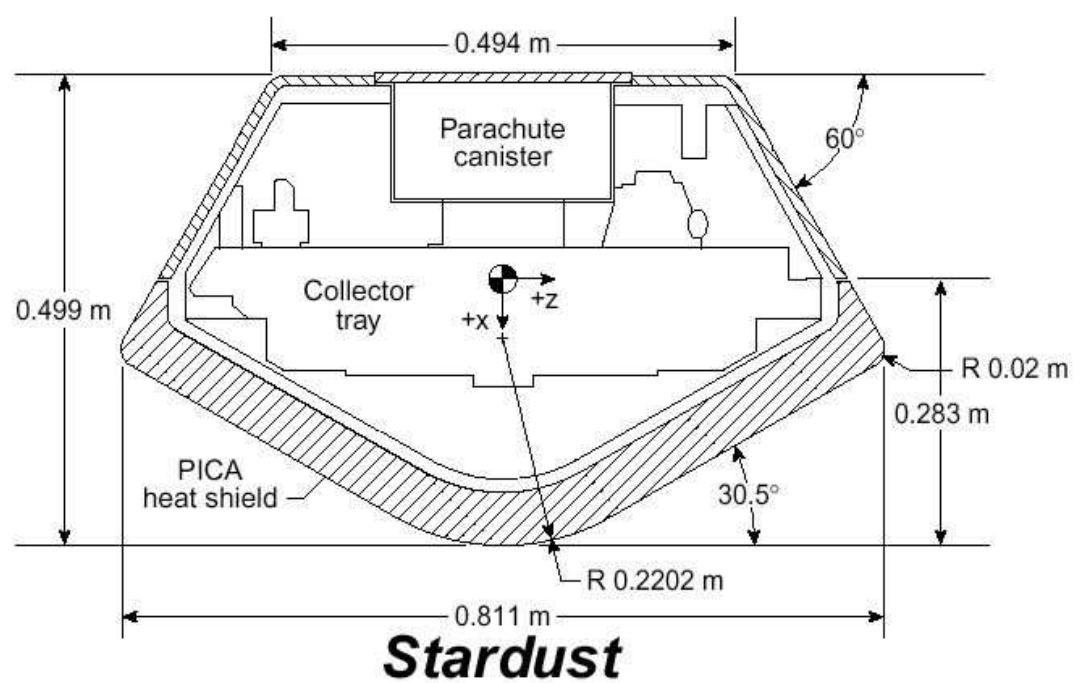

Figure 1. Geometry of the Stardust Return Capsule.

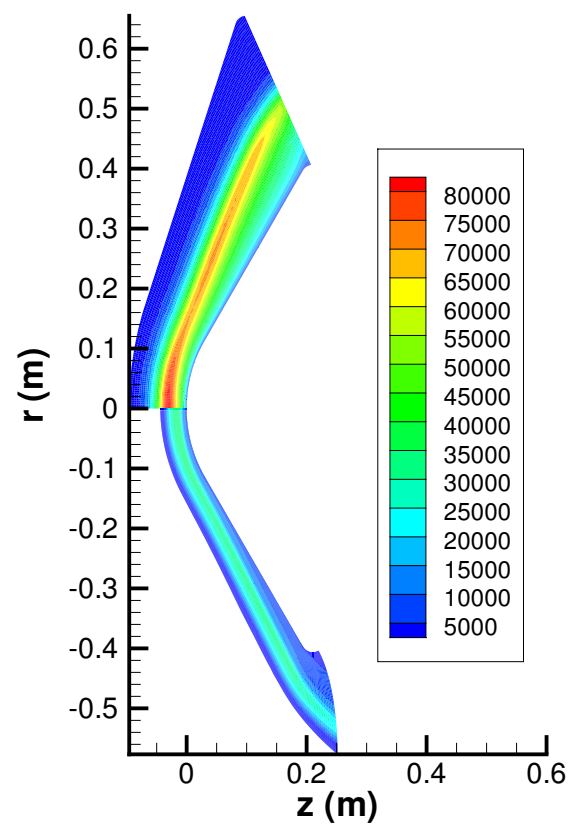

Figure 2. Contours of translational temperature (K) for the Stardust Return Capsule at $81 \mathrm{~km}$ obtained using DSMC (upper) and the baseline DPLR case (lower). 


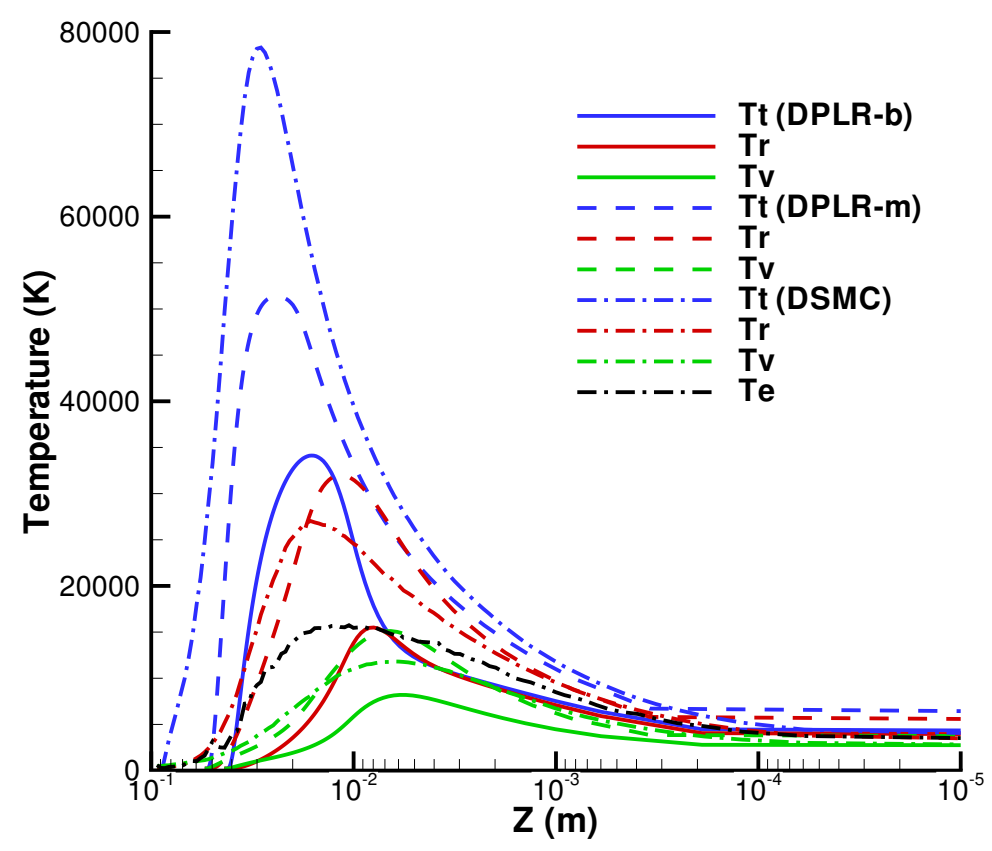

Figure 3. Profiles of temperature along the stagnation streamline for the Stardust Return Capsule at $81 \mathrm{~km}$ : DPLRb=baseline chemistry; DPLR-m=modified chemistry.

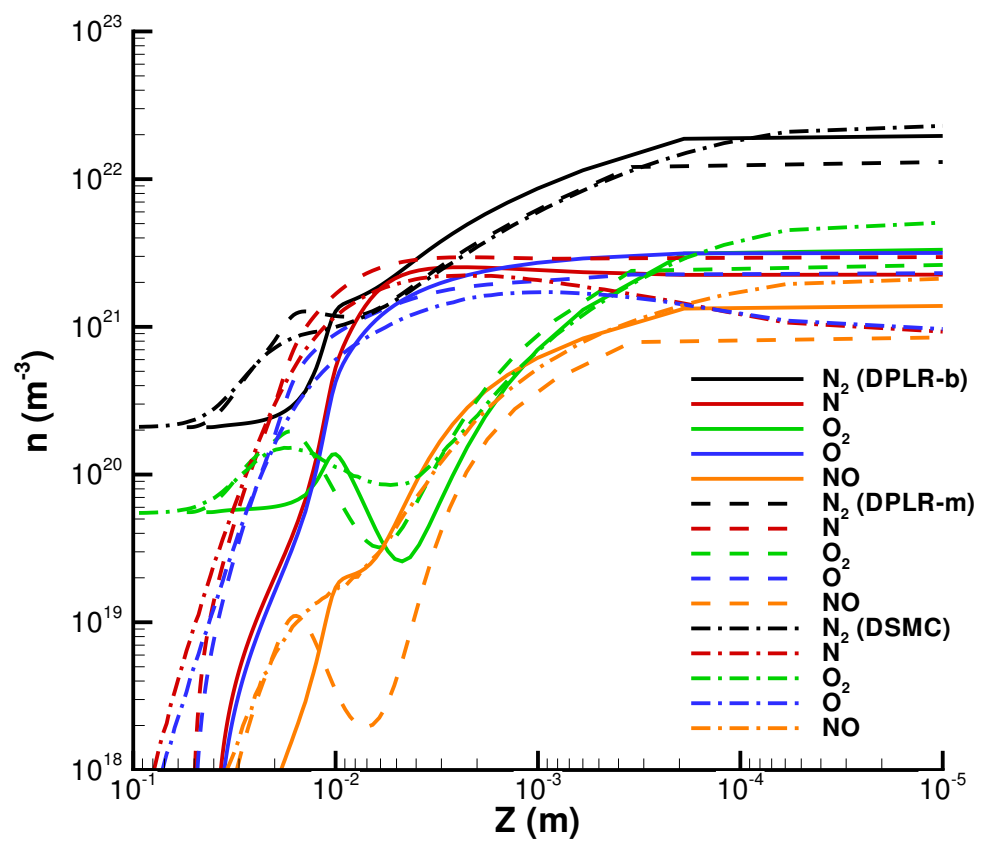

Figure 4. Profiles of neutral species number density along the stagnation streamline for the Stardust Return Capsule at $81 \mathrm{~km}$ : DPLR-b=baseline chemistry; DPLR-m=modified chemistry. 


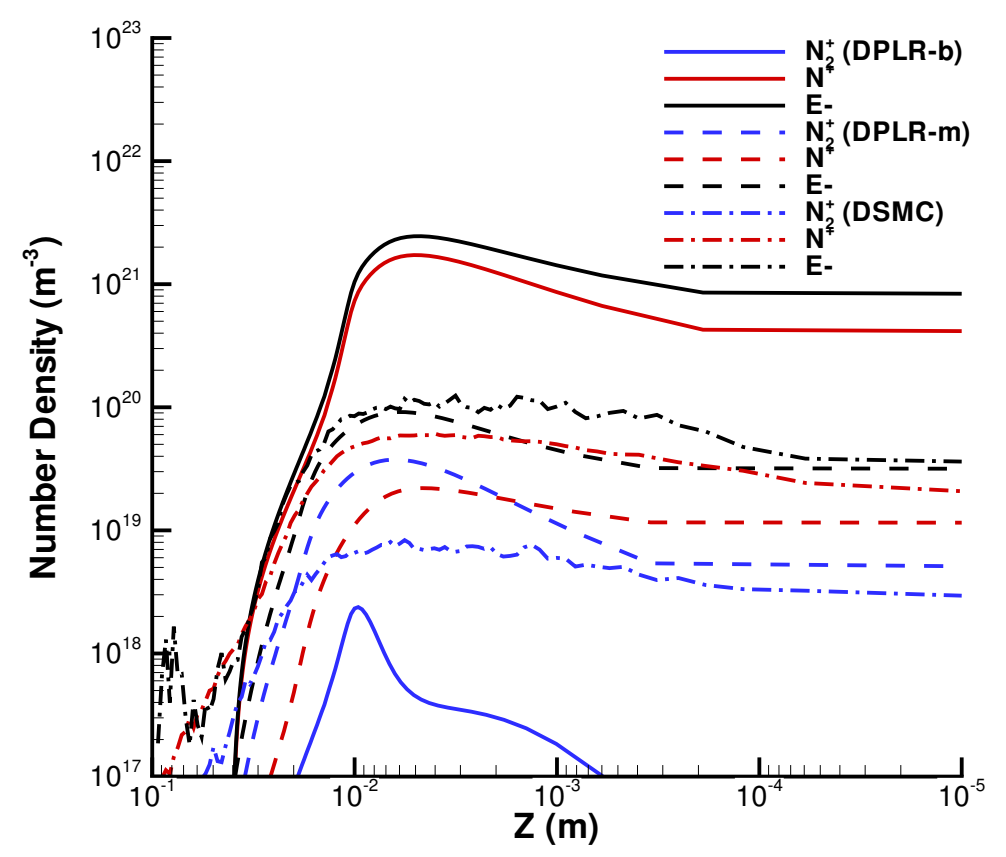

Figure 5. Profiles of charged species number density along the stagnation streamline for the Stardust Return Capsule at $81 \mathrm{~km}$ : DPLR-b=baseline chemistry; DPLR-m=modified chemistry.

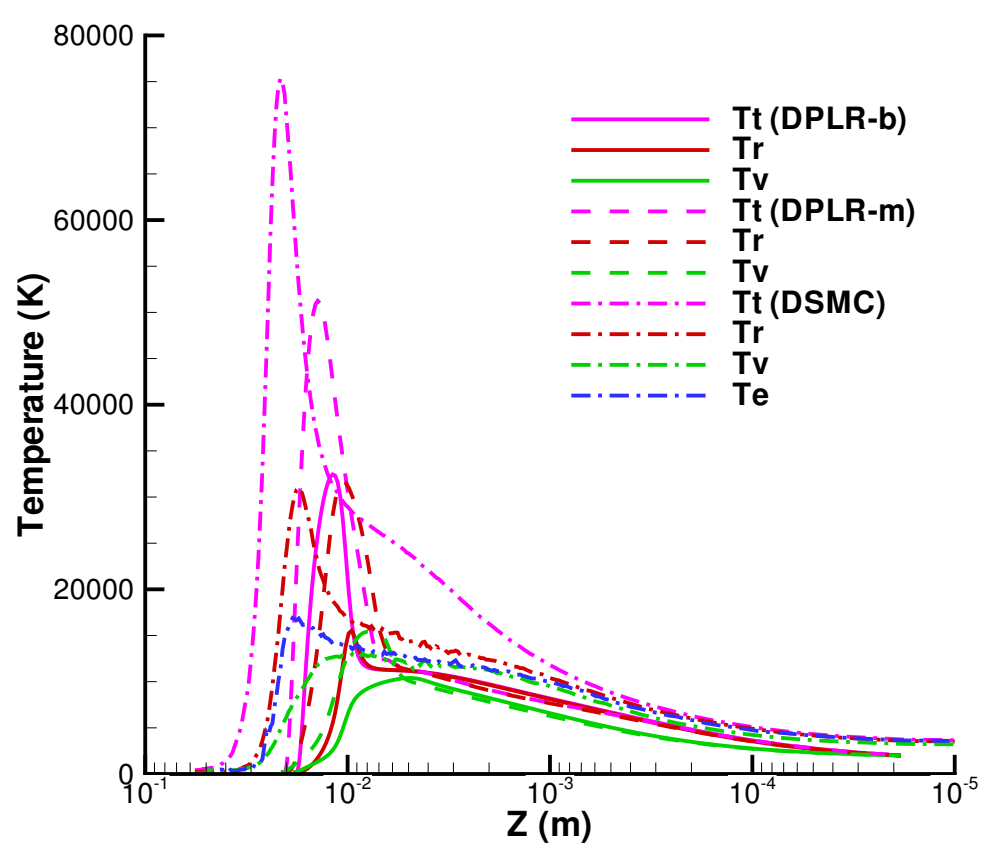

Figure 6. Profiles of temperature along the stagnation streamline for the Stardust Return Capsule at $71 \mathrm{~km}$ : DPLRb=baseline chemistry; DPLR-m=modified chemistry. 


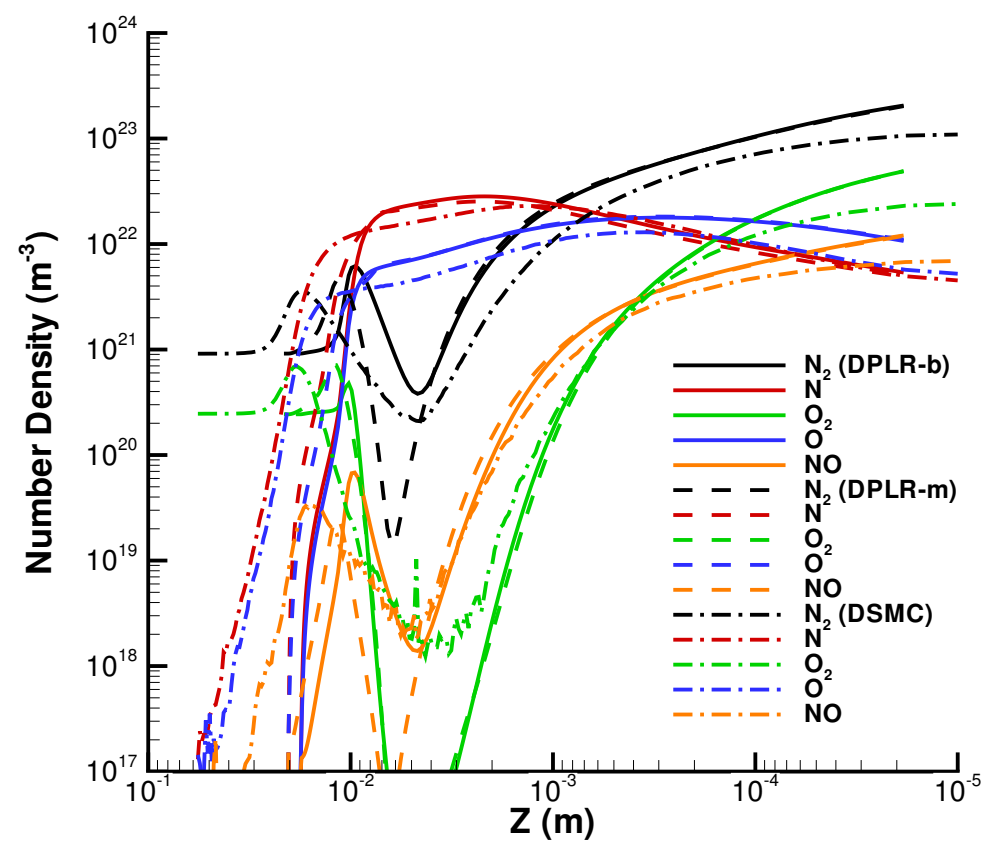

Figure 7. Profiles of neutral species number density along the stagnation streamline for the Stardust Return Capsule at $71 \mathrm{~km}$ : DPLR-b=baseline chemistry; DPLR-m=modified chemistry.

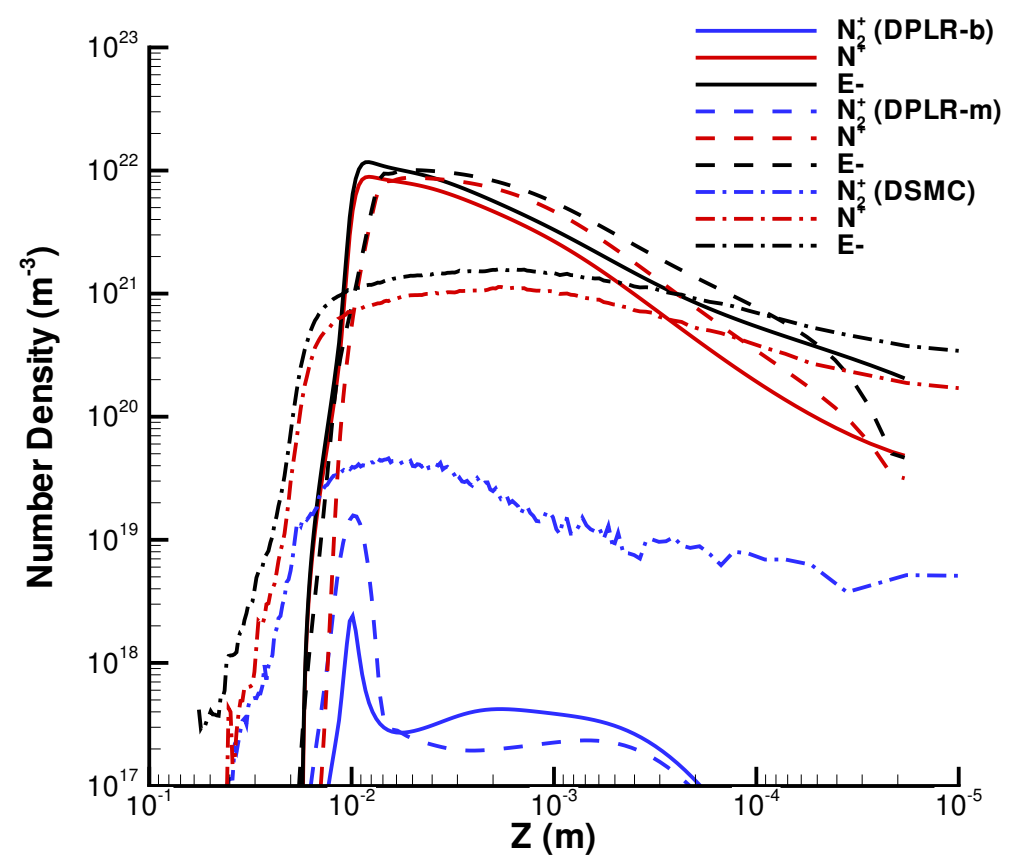

Figure 8. Profiles of charged species number density along the stagnation streamline for the Stardust Return Capsule at $71 \mathrm{~km}$ : DPLR-b=baseline chemistry; DPLR-m=modified chemistry. 


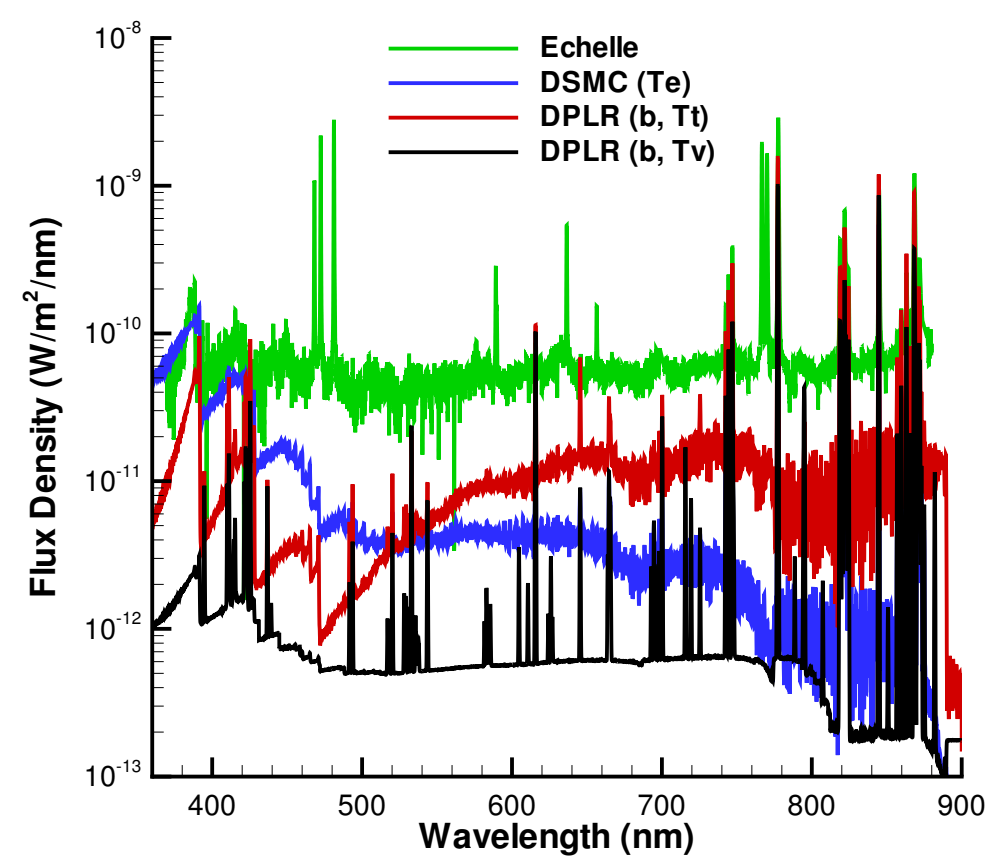

Figure 9. Comparisons of measured and computed spectra across the full range of the Echelle instrument at 81 km: DPLR-b=baseline chemistry.

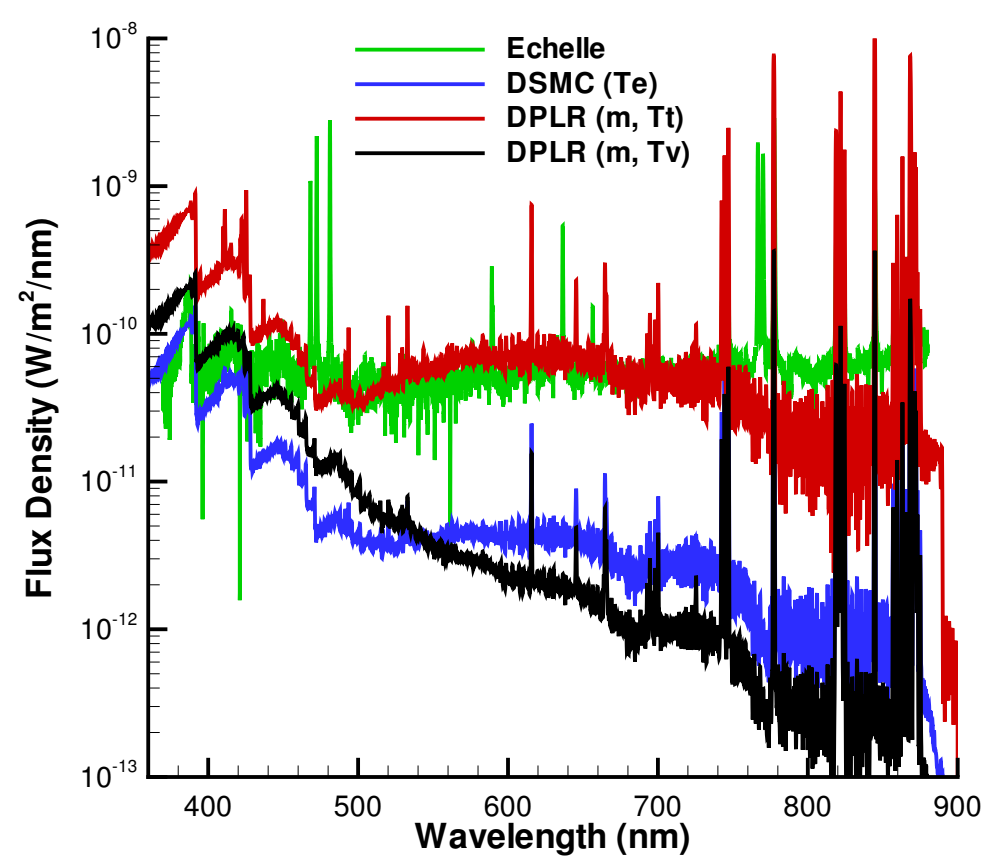

Figure 10. Comparisons of measured and computed spectra across the full range of the Echelle instrument at 81 km: DPLR-m=modified chemistry. 


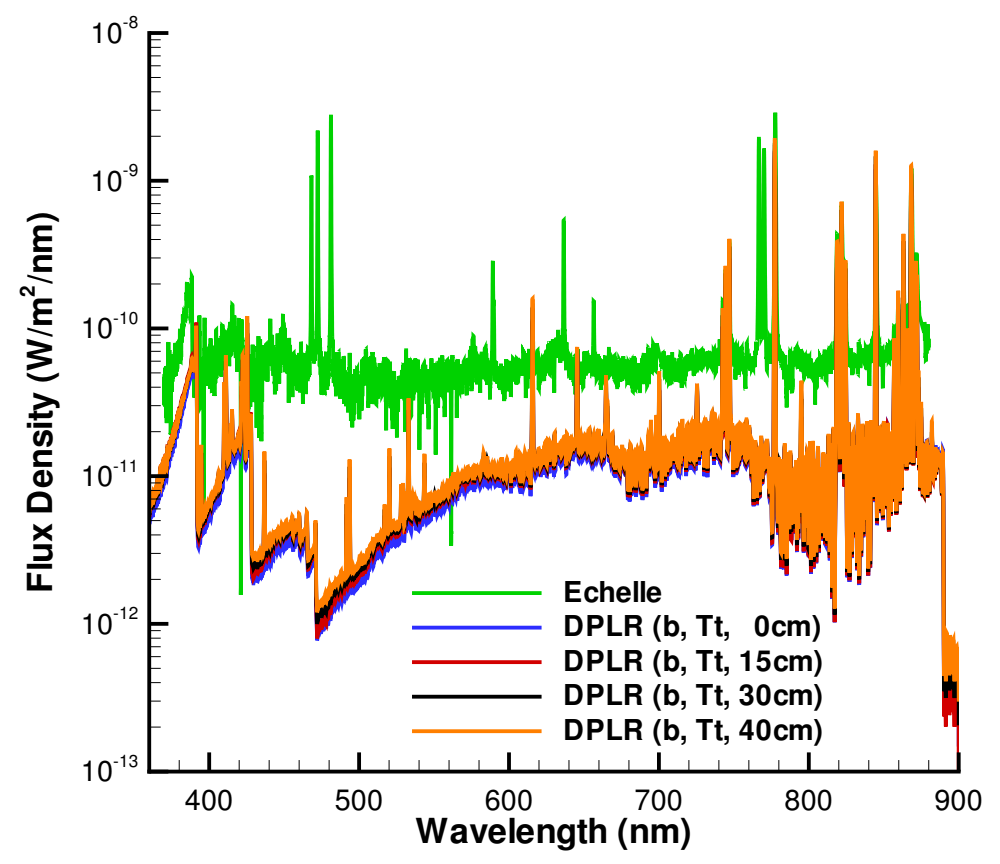

Figure 11. Radial variation of computed spectra across the full range of the Echelle instrument at $81 \mathrm{~km}$ : DPLRb=baseline chemistry.

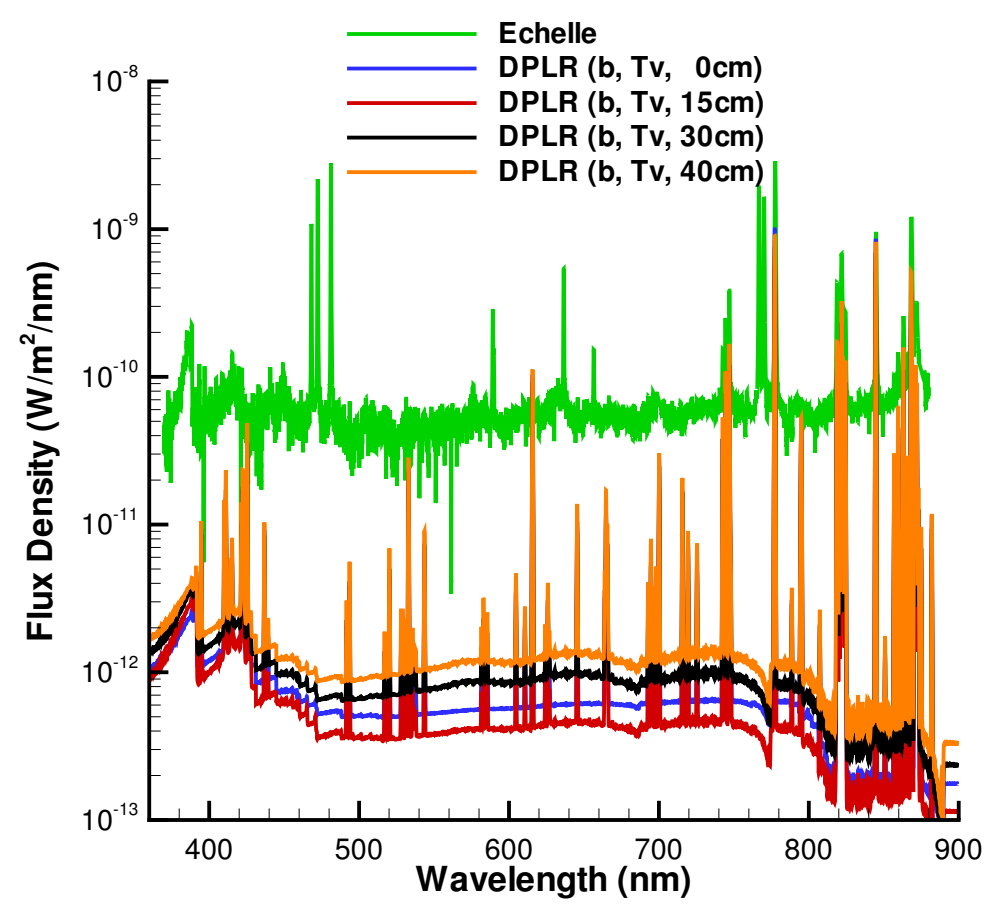

Figure 12. Radial variation of computed spectra across the full range of the Echelle instrument at $81 \mathrm{~km}$ : DPLRb=baseline chemistry. 


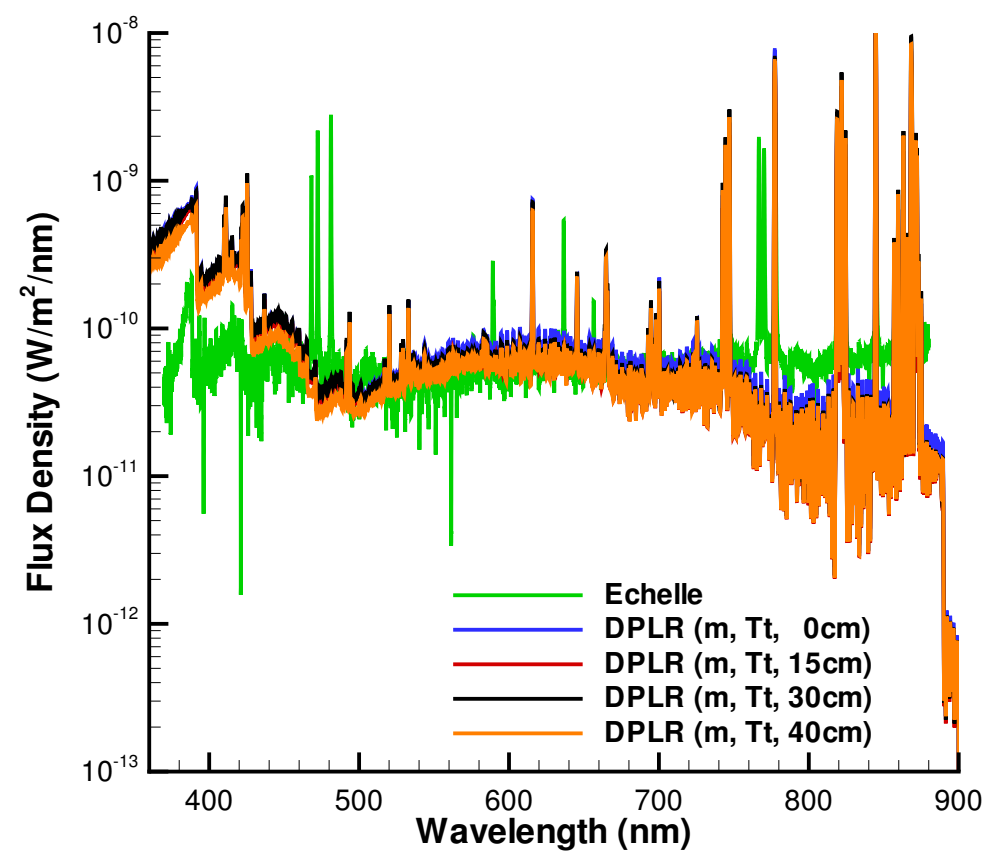

Figure 13. Radial variation of computed spectra across the full range of the Echelle instrument at $81 \mathrm{~km}$ : DPLRm=modified chemistry.

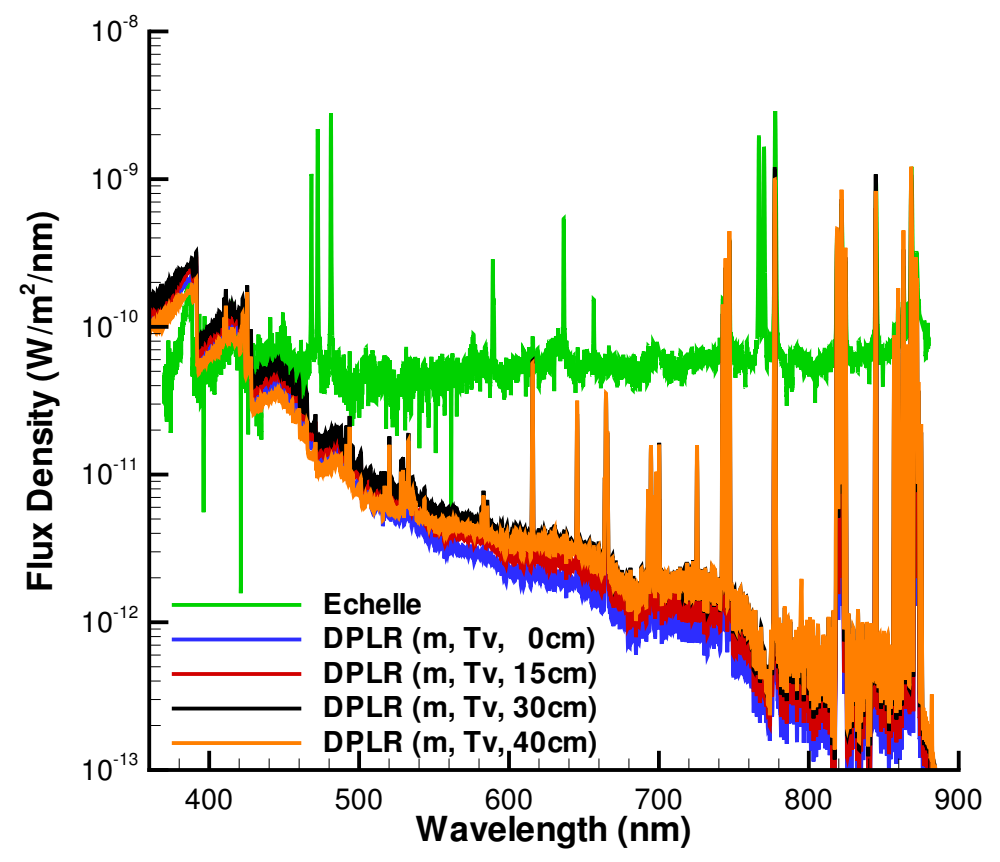

Figure 14. Radial variation of computed spectra across the full range of the Echelle instrument at $81 \mathrm{~km}$ : DPLRm=modified chemistry. 


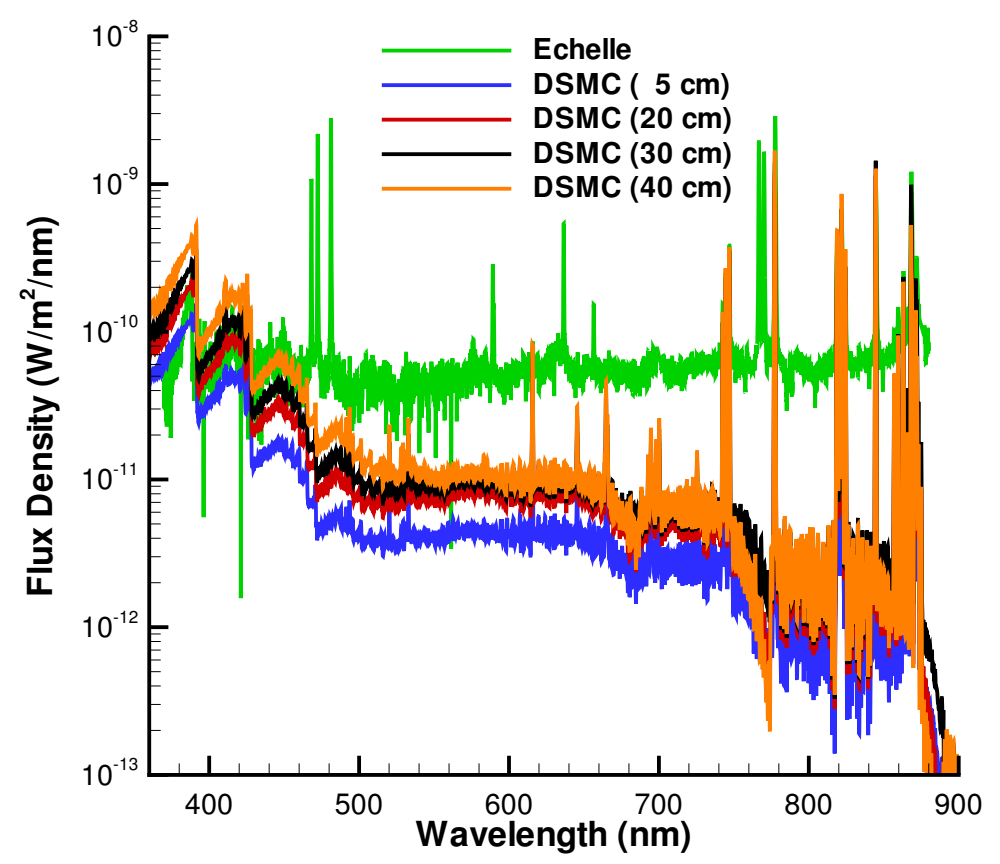

Figure 15. Radial variation of computed spectra across the full range of the Echelle instrument at $81 \mathrm{~km}$.

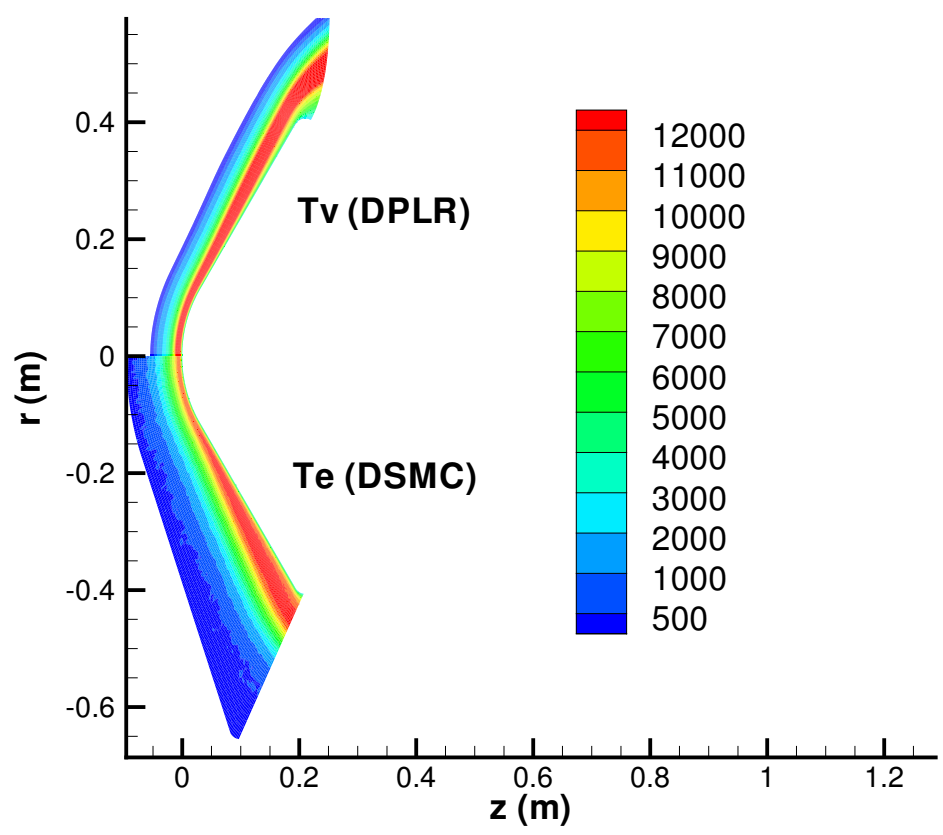

Figure 16. Contours of vibrational temperature (K) obtained using the modified DPLR case (upper) and contours of electron temperature from DSMC (lower) at $81 \mathrm{~km}$. 


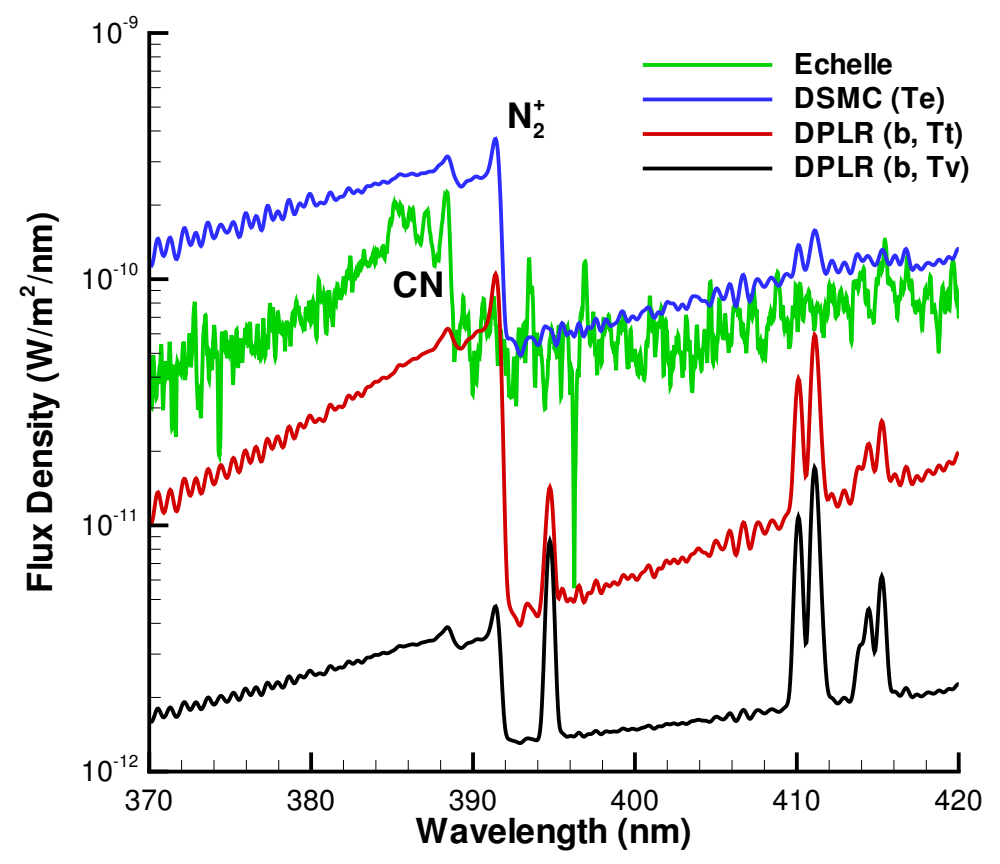

Figure 17. Comparisons of measured and computed spectra focusing on air molecular bands at $81 \mathrm{~km}$ : DPLR-b=baseline chemistry.

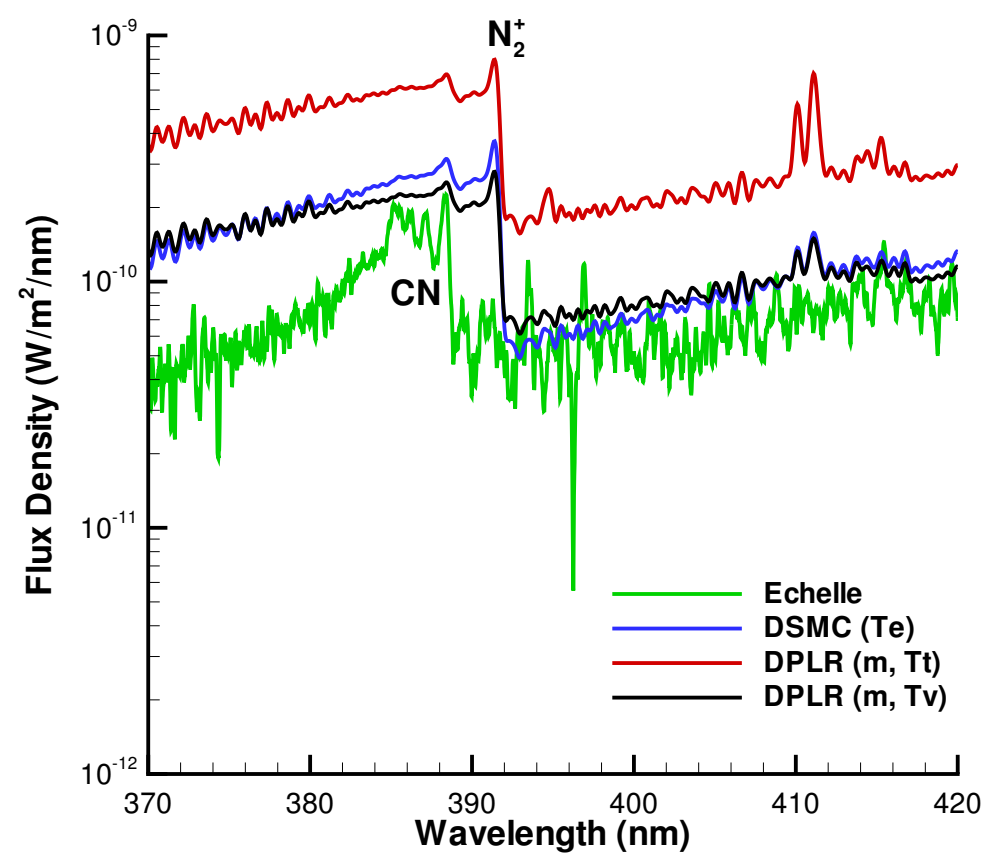

Figure 18. Comparisons of measured and computed spectra focusing on air molecular bands at 81 km: DPLRm=modified chemistry. 


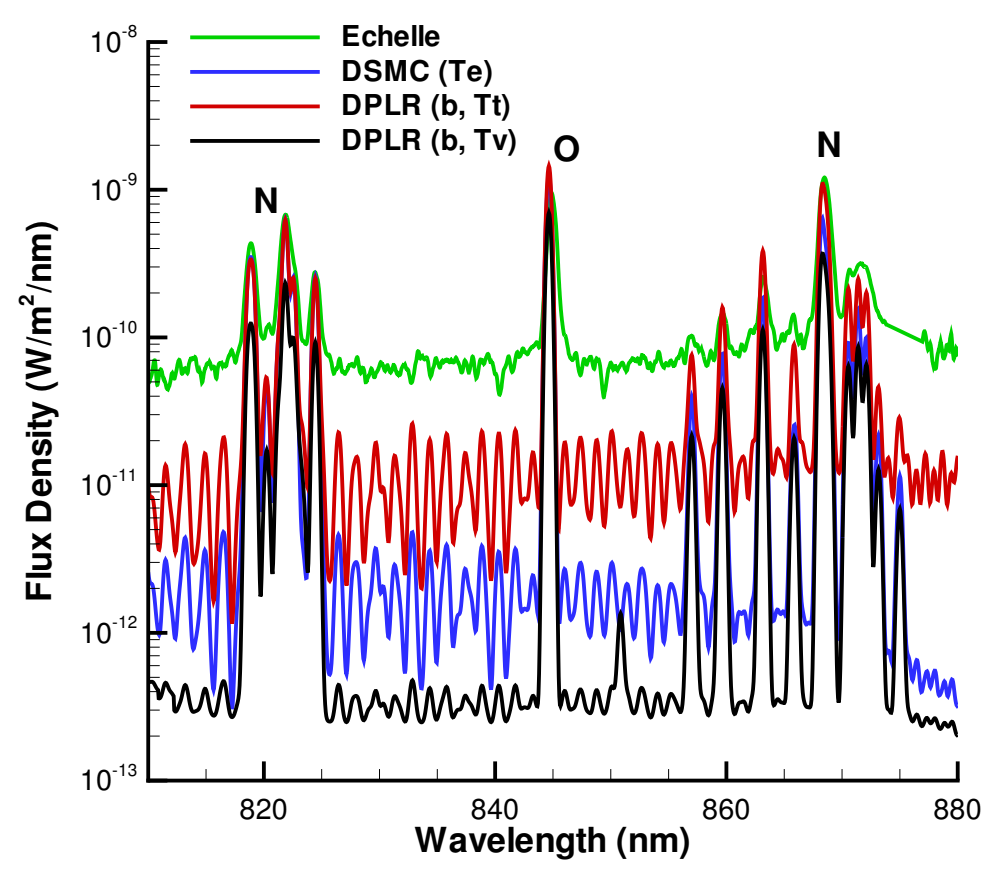

Figure 19. Comparisons of measured and computed spectra focusing on air atomic lines at $81 \mathrm{~km}$ : DPLR-b=baseline chemistry.

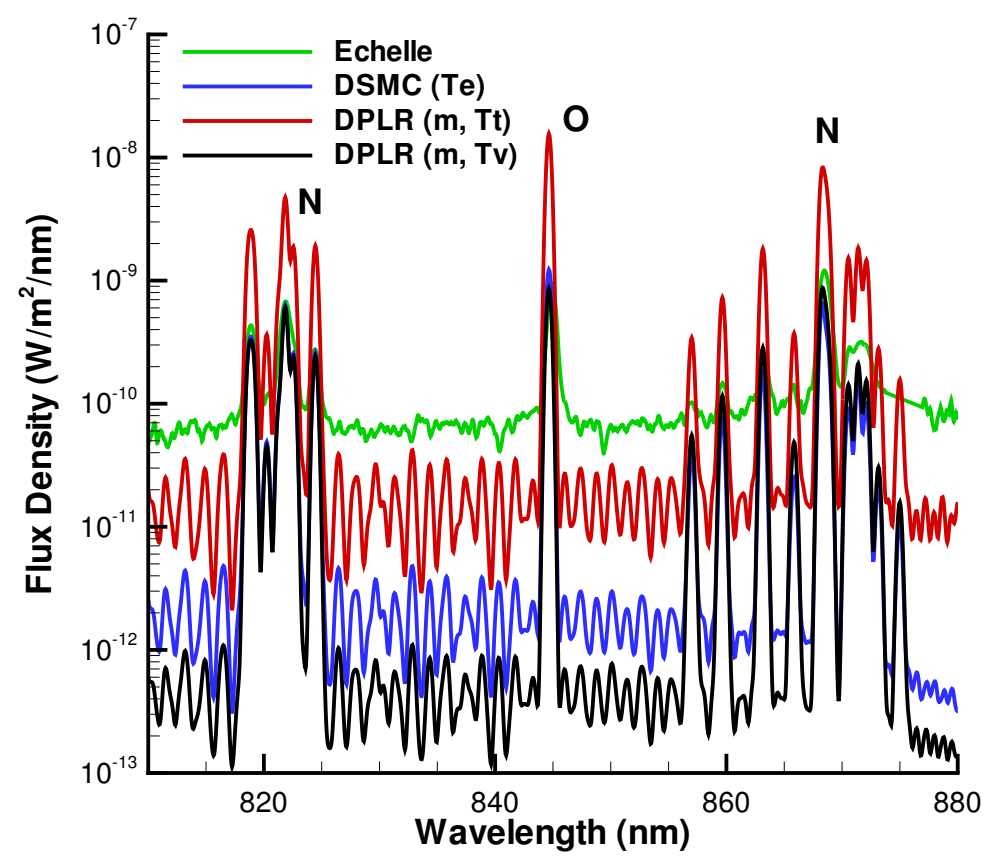

Figure 20. Comparisons of measured and computed spectra focusing on air atomic lines at $81 \mathrm{~km}$ : DPLR-m=modified chemistry. 


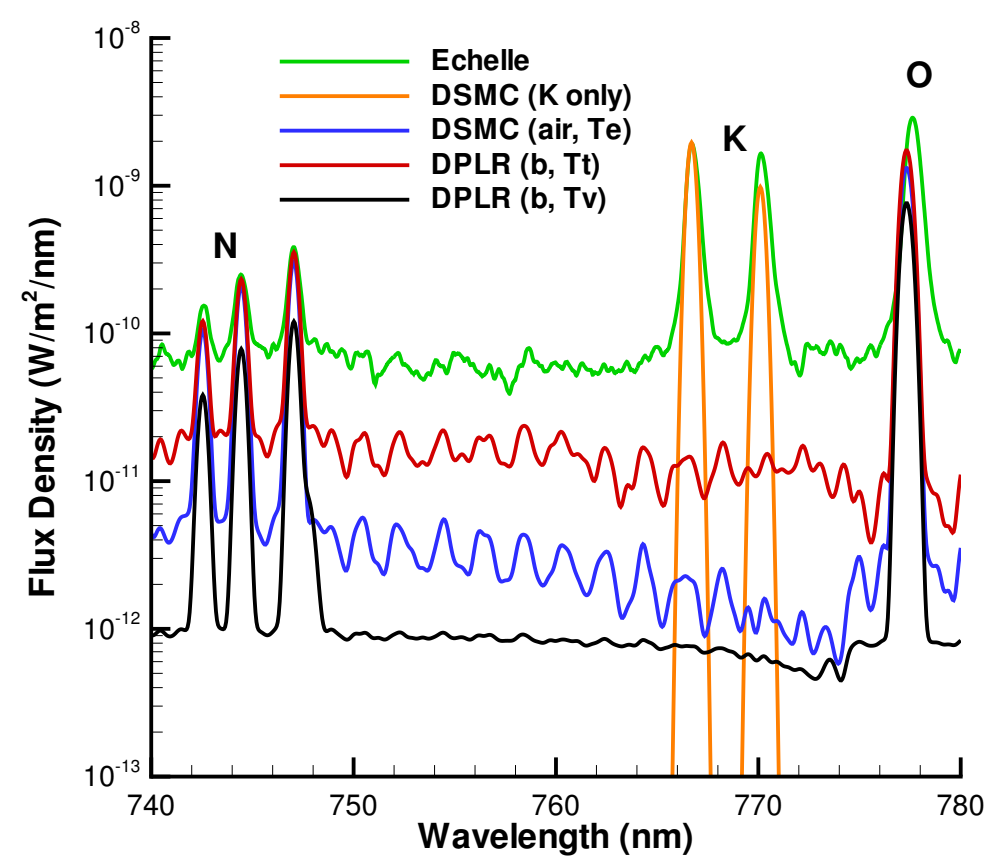

Figure 21. Comparisons of measured and computed spectra focusing on air and potassium atomic lines at 81 km: DPLR-b=baseline chemistry.

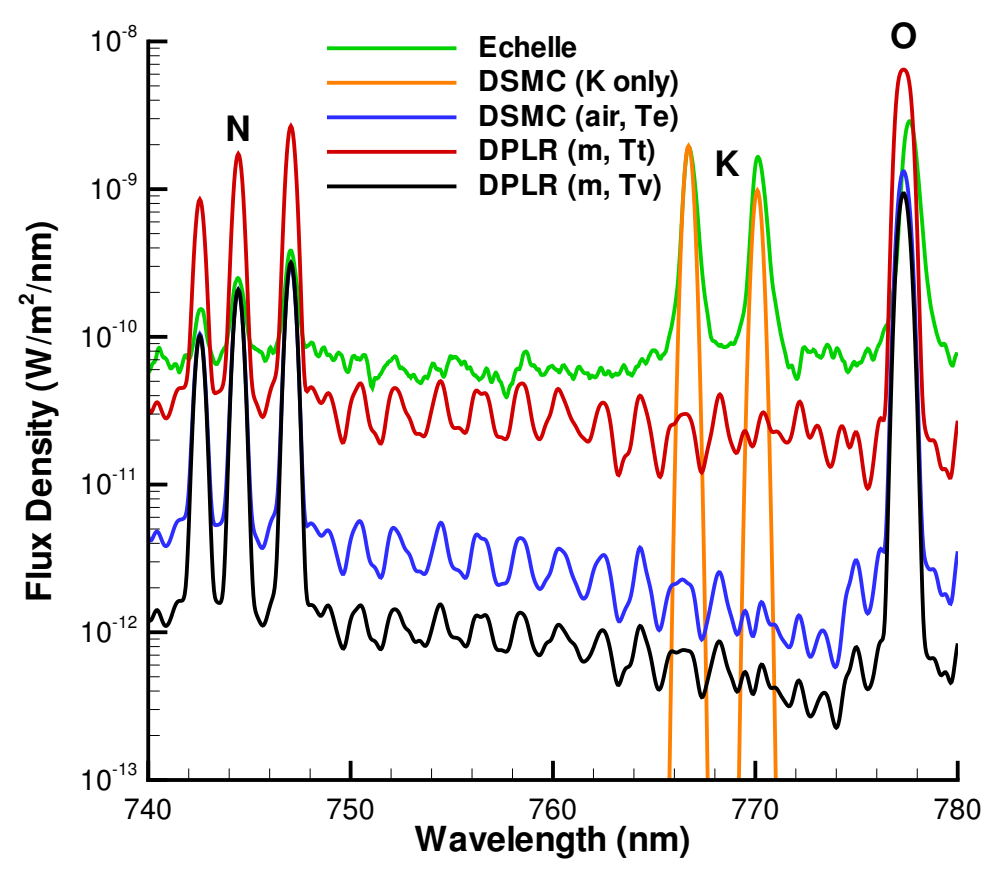

Figure 22. Comparisons of measured and computed spectra focusing on air and potassium atomic lines at 81 km: DPLR-m=modified chemistry. 


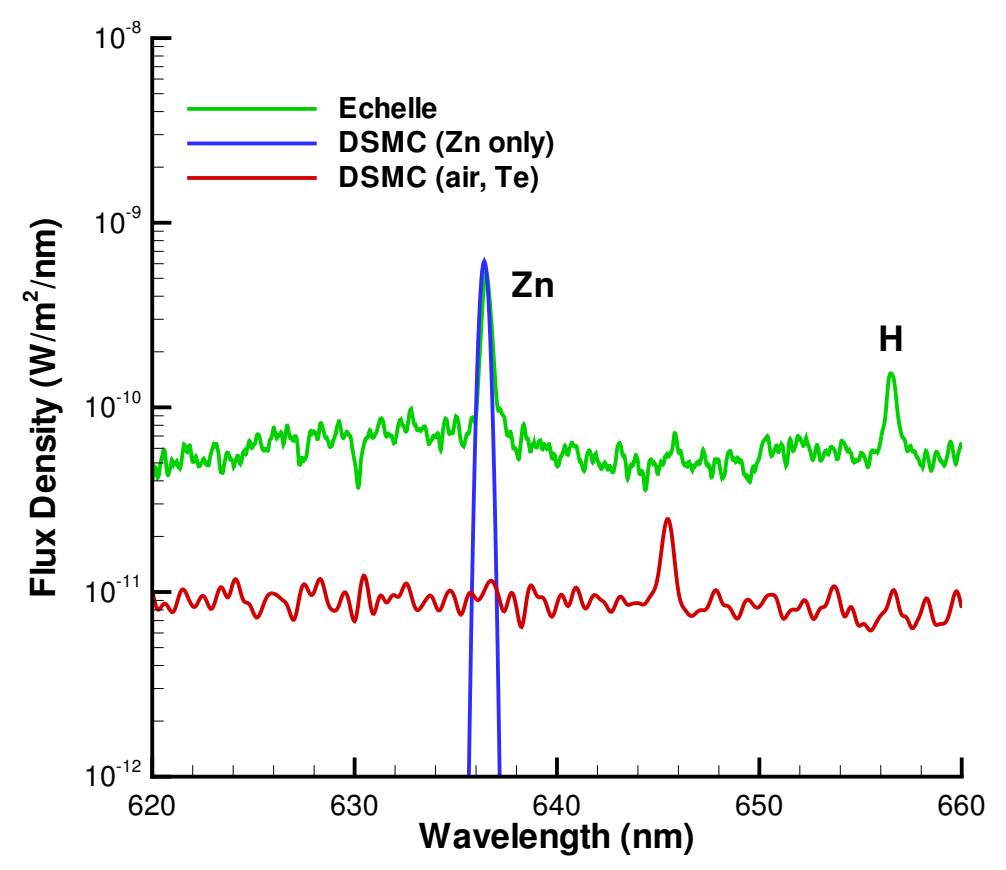

Figure 23. Comparisons of measured and computed spectra focusing on a zinc atomic line at $81 \mathrm{~km}$.

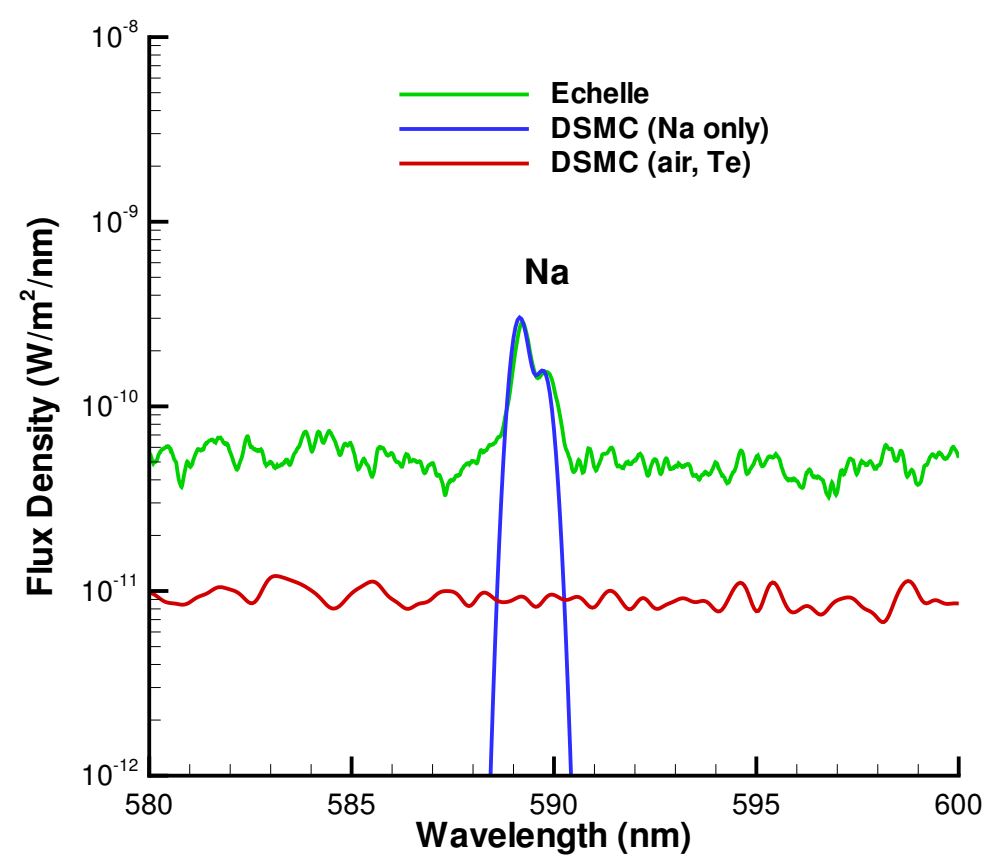

Figure 24. Comparisons of measured and computed spectra focusing on sodium atomic lines at $81 \mathrm{~km}$. 


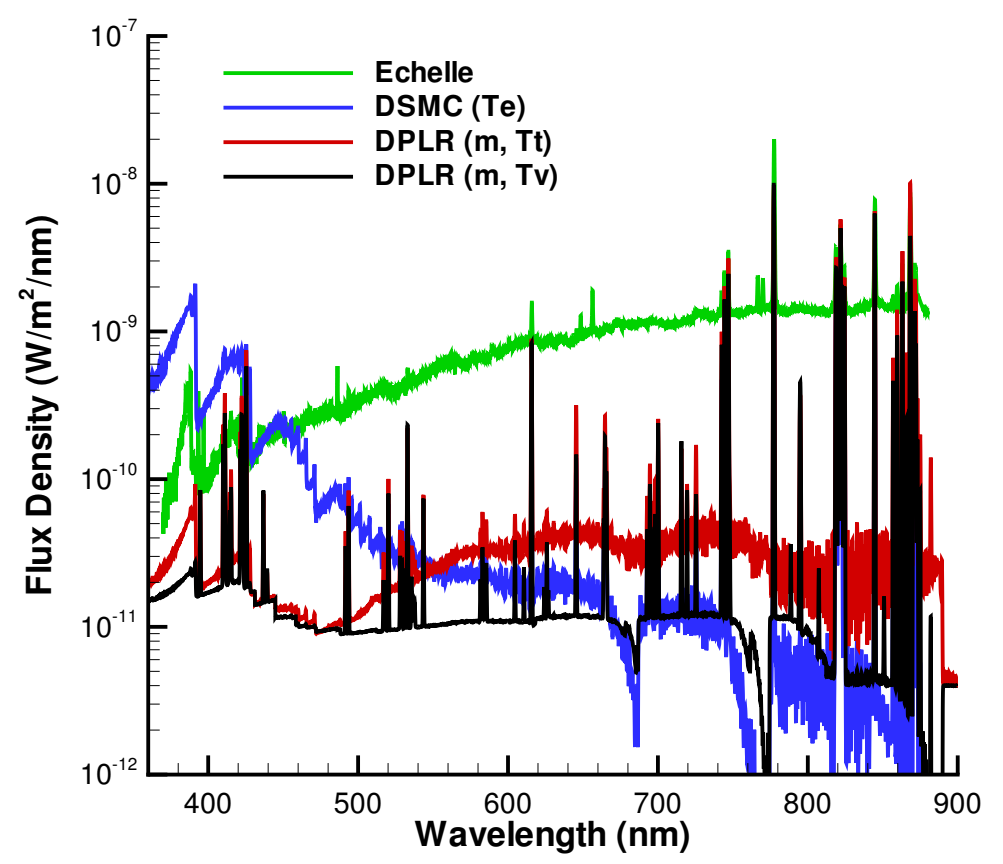

Figure 25. Comparisons of measured and computed spectra across the full range of the Echelle instrument at $71 \mathrm{~km}$ : DPLR-m $=$ modified chemistry.

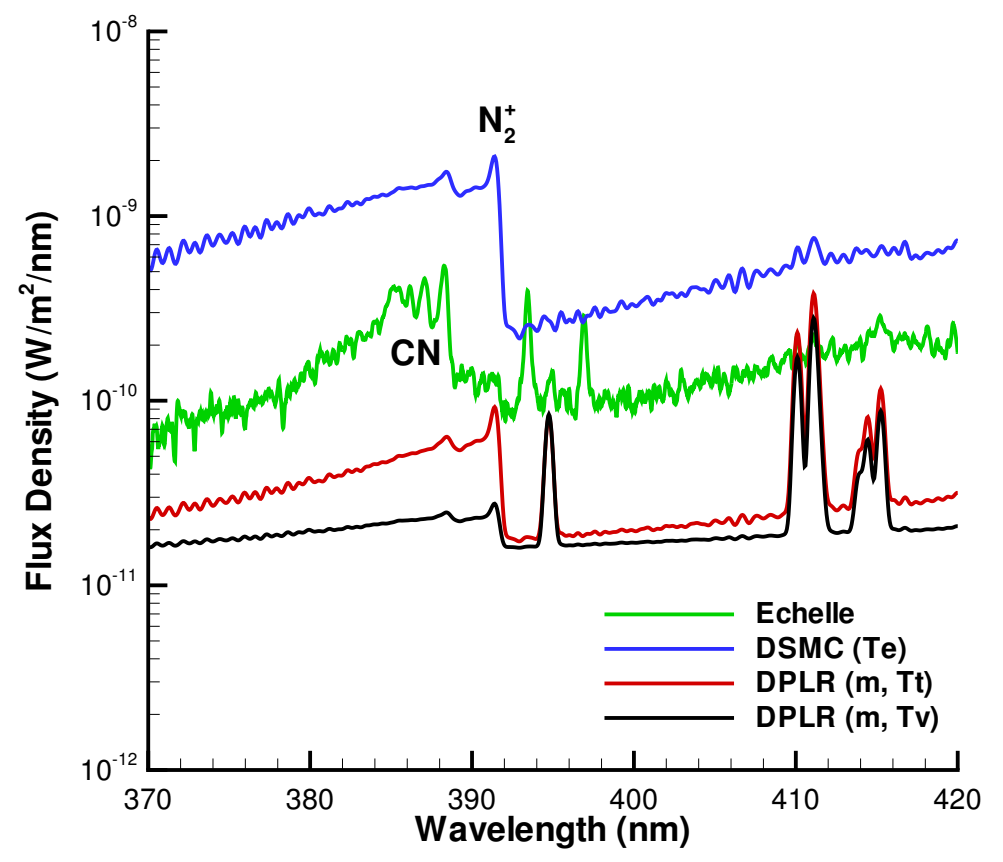

Figure 26. Comparisons of measured and computed spectra at $71 \mathrm{~km}$ focusing on air molecular bands: DPLRm=modified chemistry. 


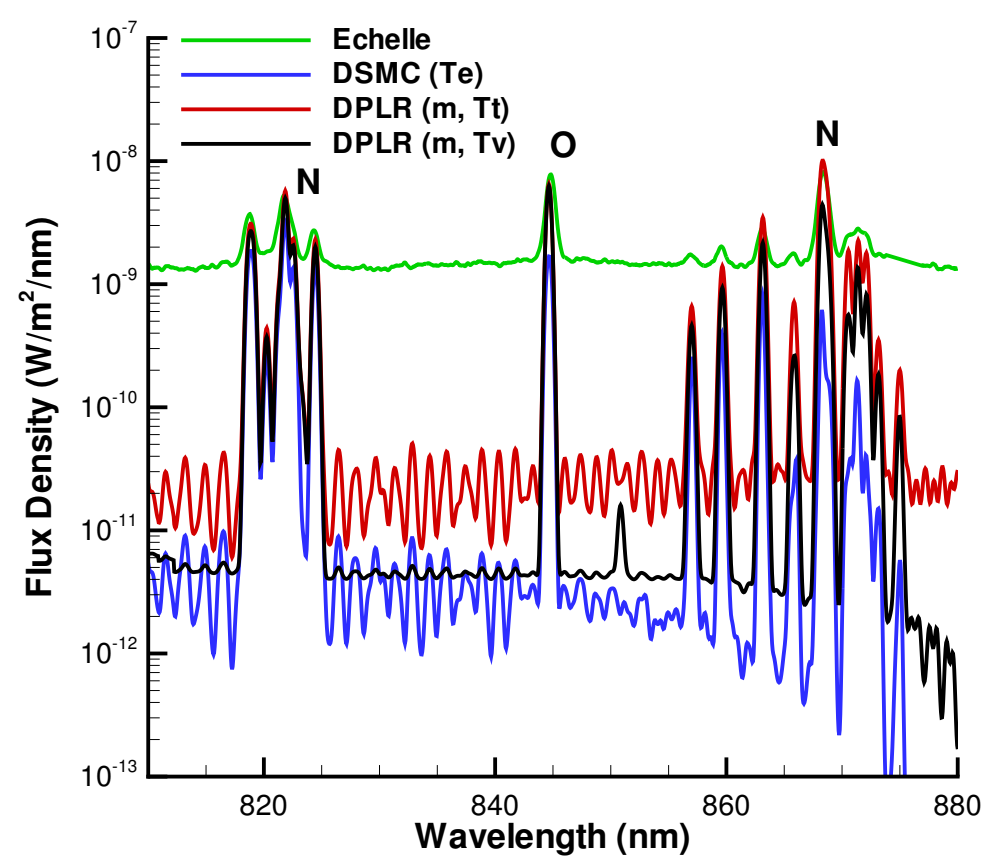

Figure 27. Comparisons of measured and computed spectra at $71 \mathrm{~km}$ focusing on air atomic lines: DPLR-m=modified chemistry.

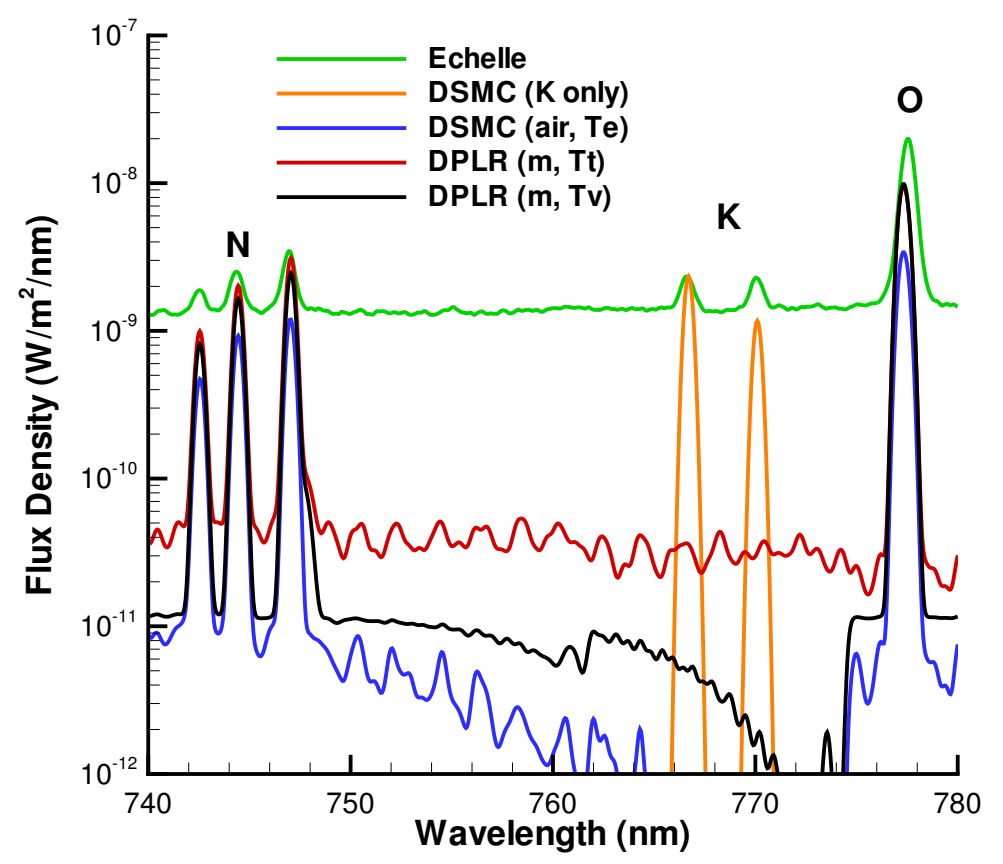

Figure 28. Comparisons of measured and computed spectra at $71 \mathrm{~km}$ focusing on air and potassium atomic lines: DPLR-m $=$ modified chemistry. 


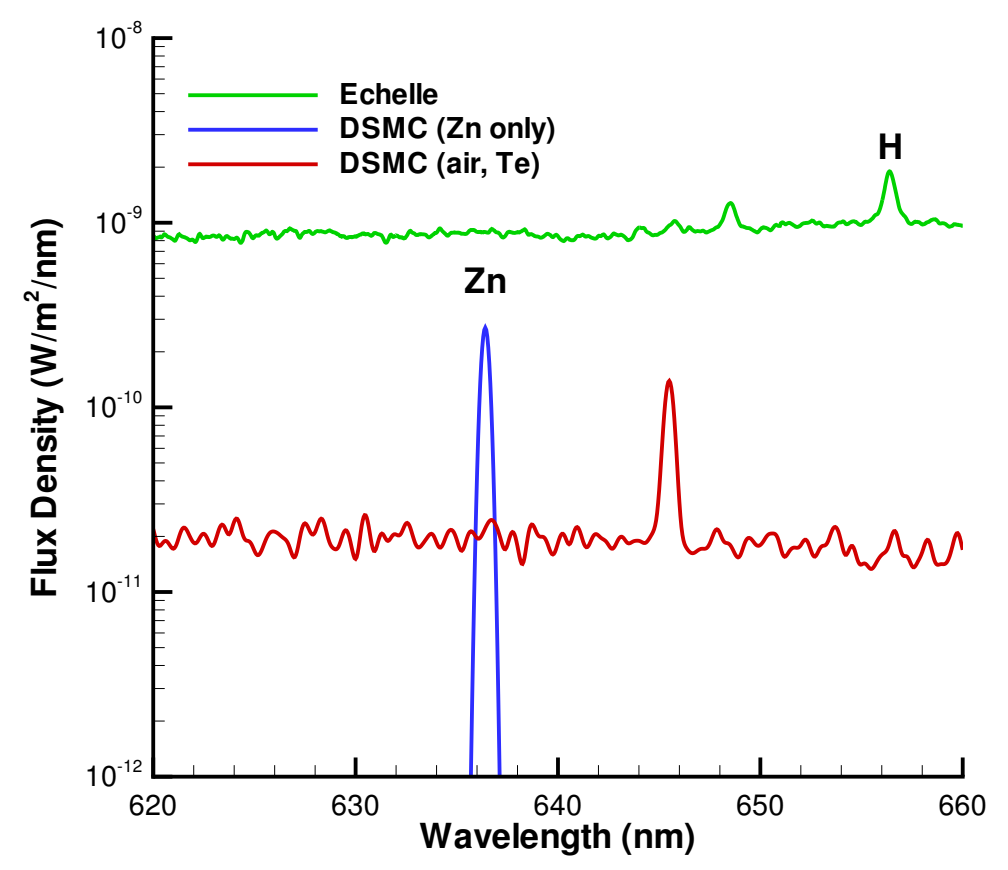

Figure 29. Comparisons of measured and computed spectra at $71 \mathrm{~km}$ focusing on a zinc atomic line.

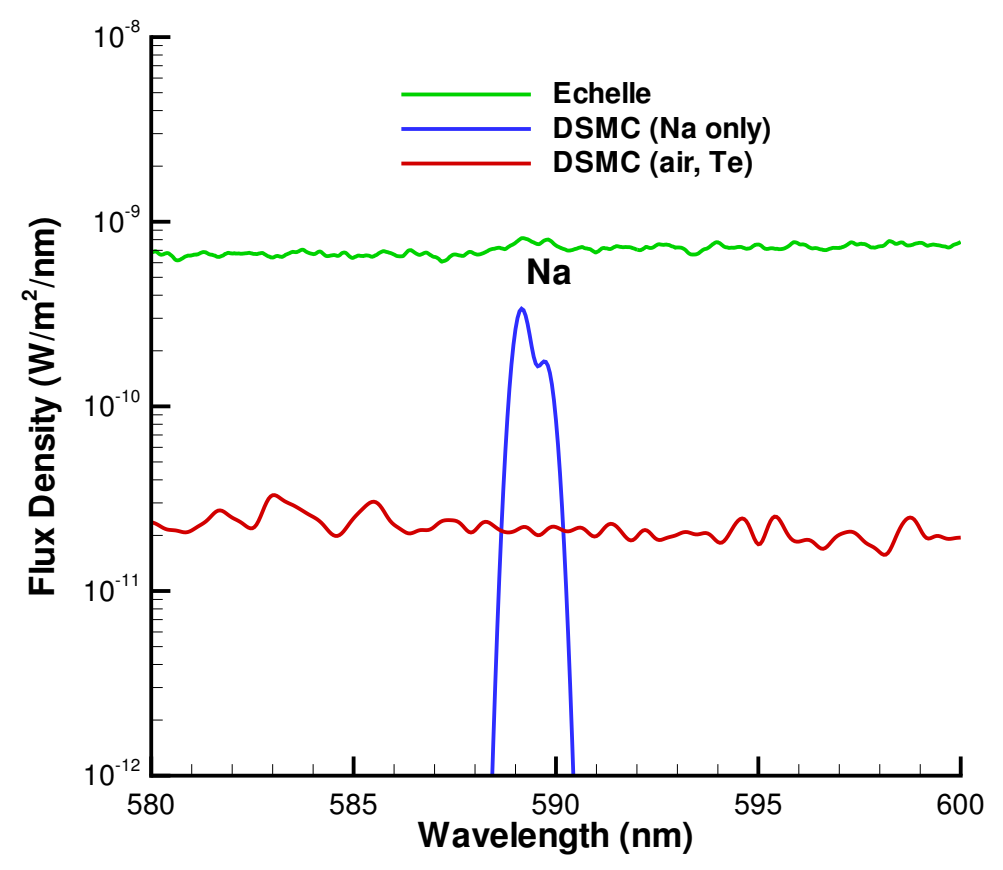

Figure 30. Comparisons of measured and computed spectra at $71 \mathrm{~km}$ focusing on sodium atomic lines. 

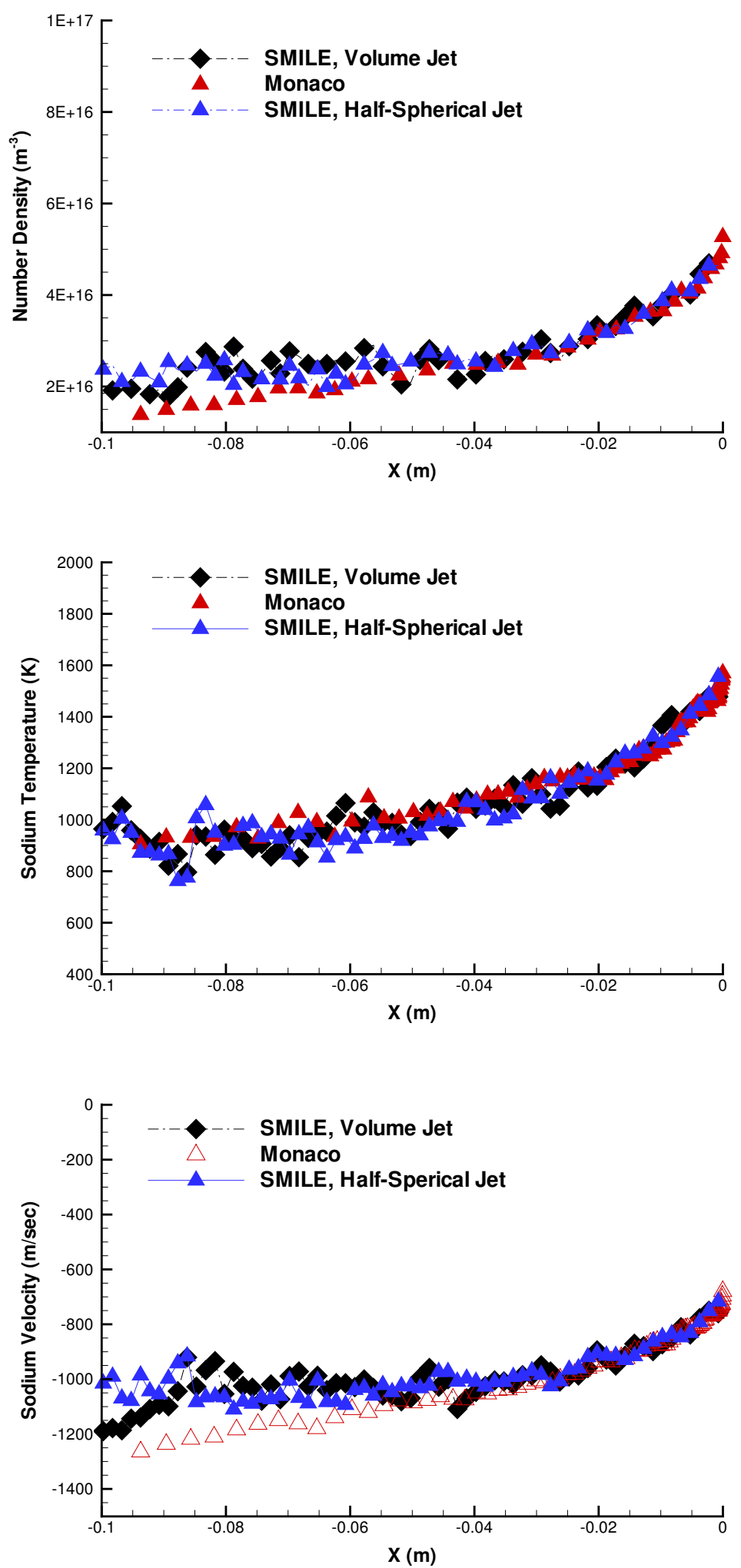

Figure 31. Comparison of sodium number density (top), temperature (middle), and velocity (bottom) along forebody stagnation line under the condition of without freestream flow. 

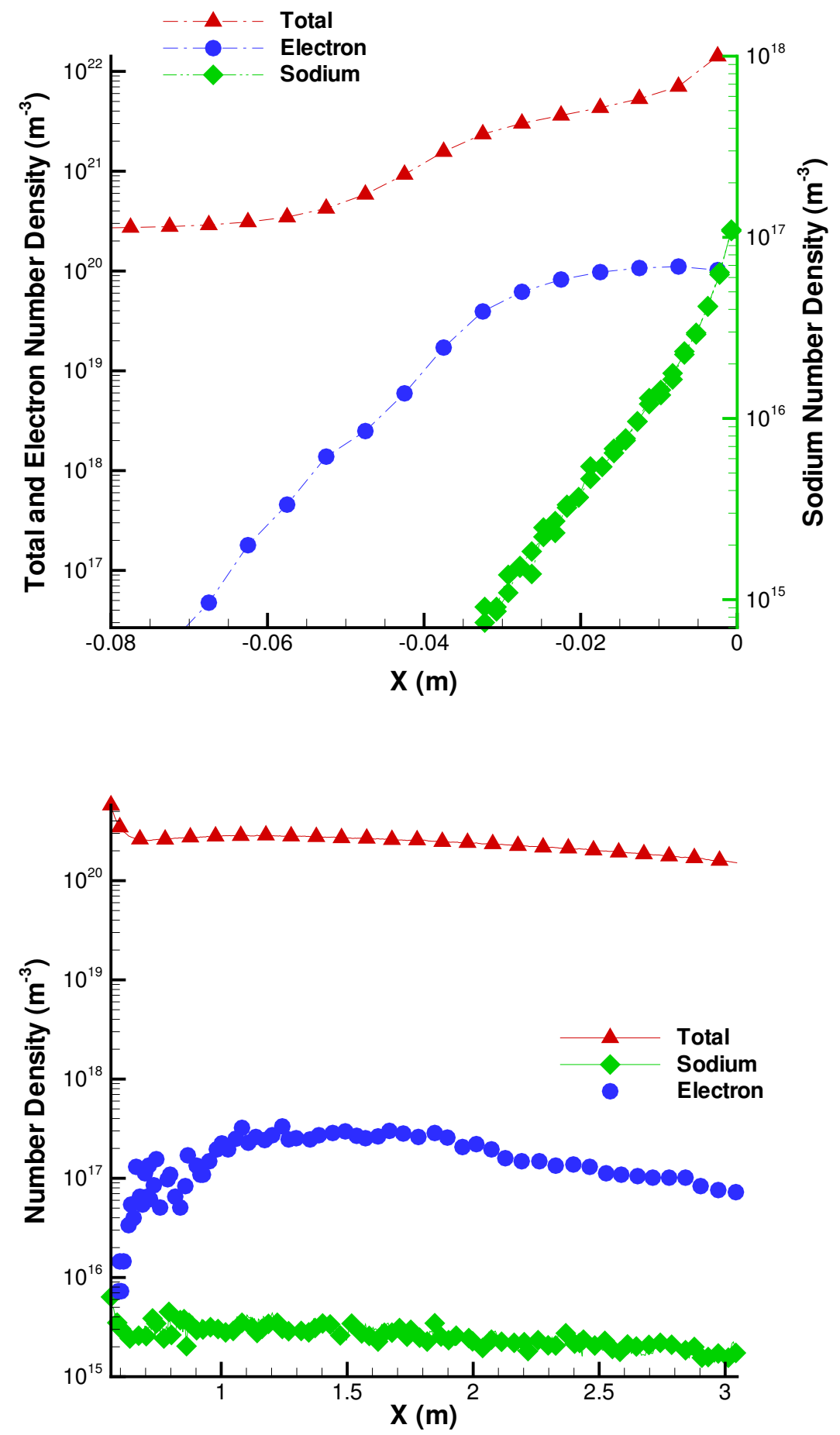

Figure 32. DSMC Solution of total, sodium and electron number densities along forebody (top) and afterbody-wake (bottom) stagnation line at $81 \mathrm{~km}$. The base of the vehicle is located at $x=0.557 \mathrm{~m}$ 

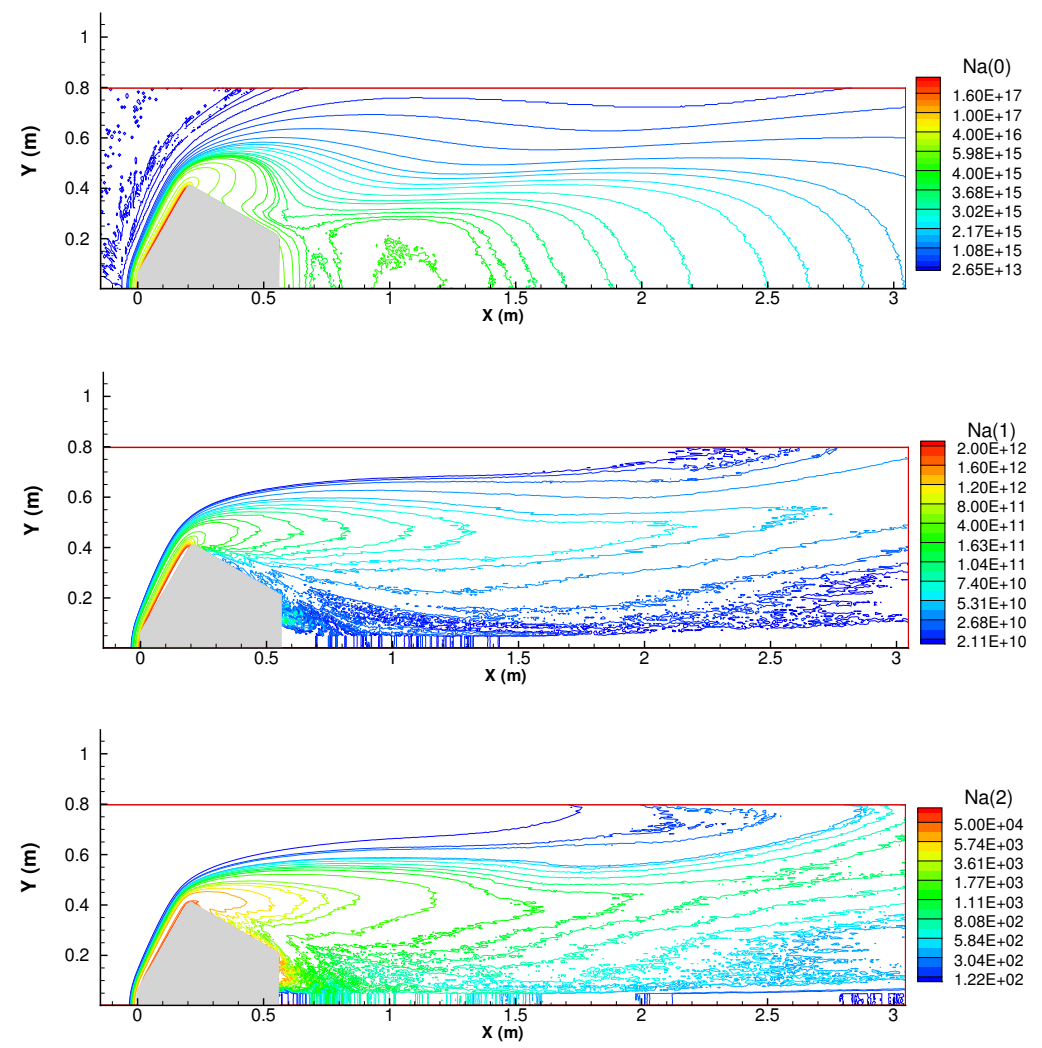

Figure 33. Number density contours of sodium atoms at the ground (top), and excitation states of ${ }^{2} \mathbf{P}_{1 / 2}$ (middle) and ${ }^{2} \mathbf{D}_{5 / 2}$ (bottom) at $81 \mathrm{~km}$. 

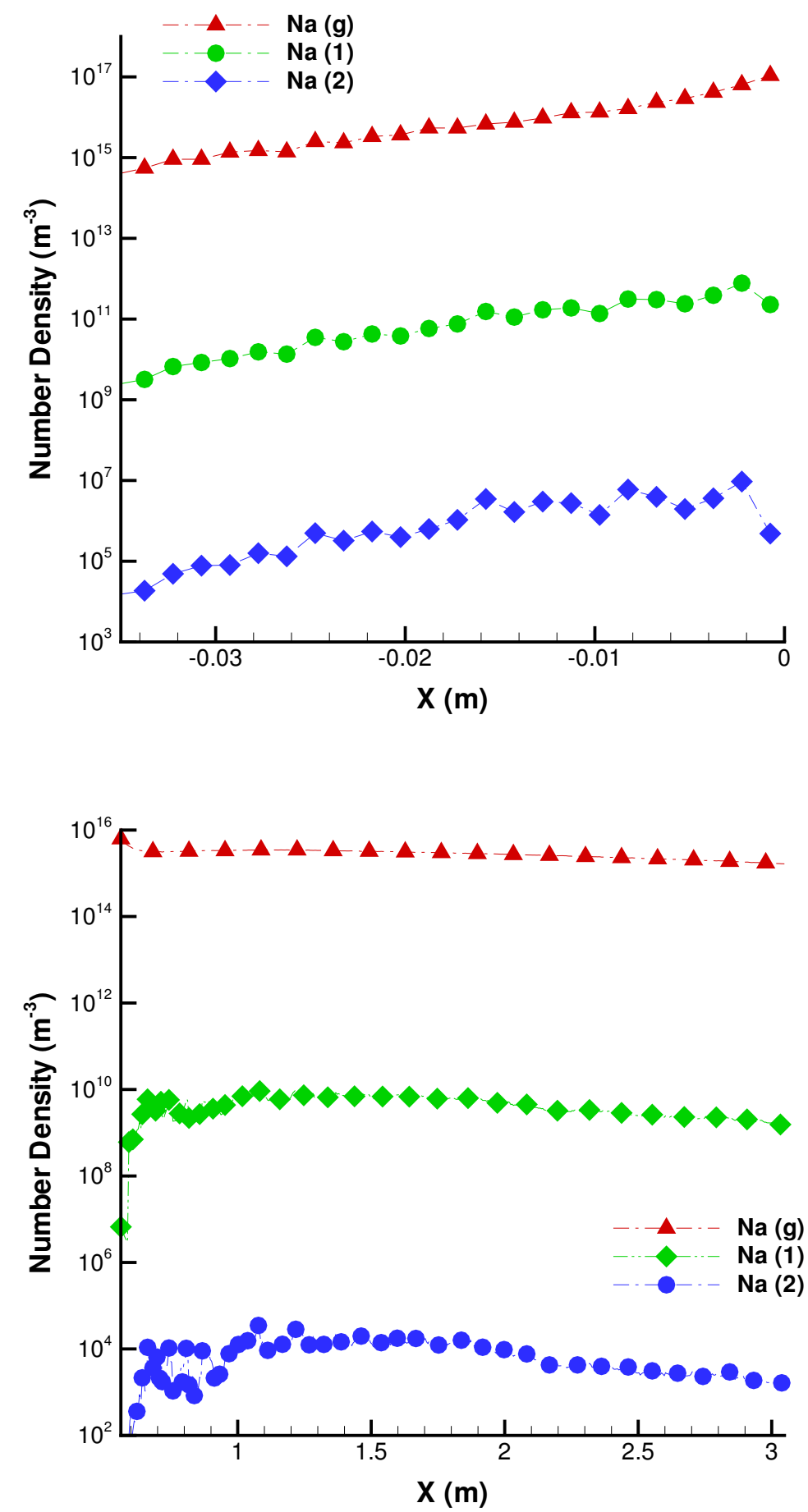

Figure 34. The distribution of sodium number density at ground, ${ }^{2} \mathbf{P}_{1 / 2}$ and ${ }^{2} \mathbf{D}_{5 / 2}$ states along forebody (top) and afterbody (bottom) stagnation line at $81 \mathrm{Km}$. 

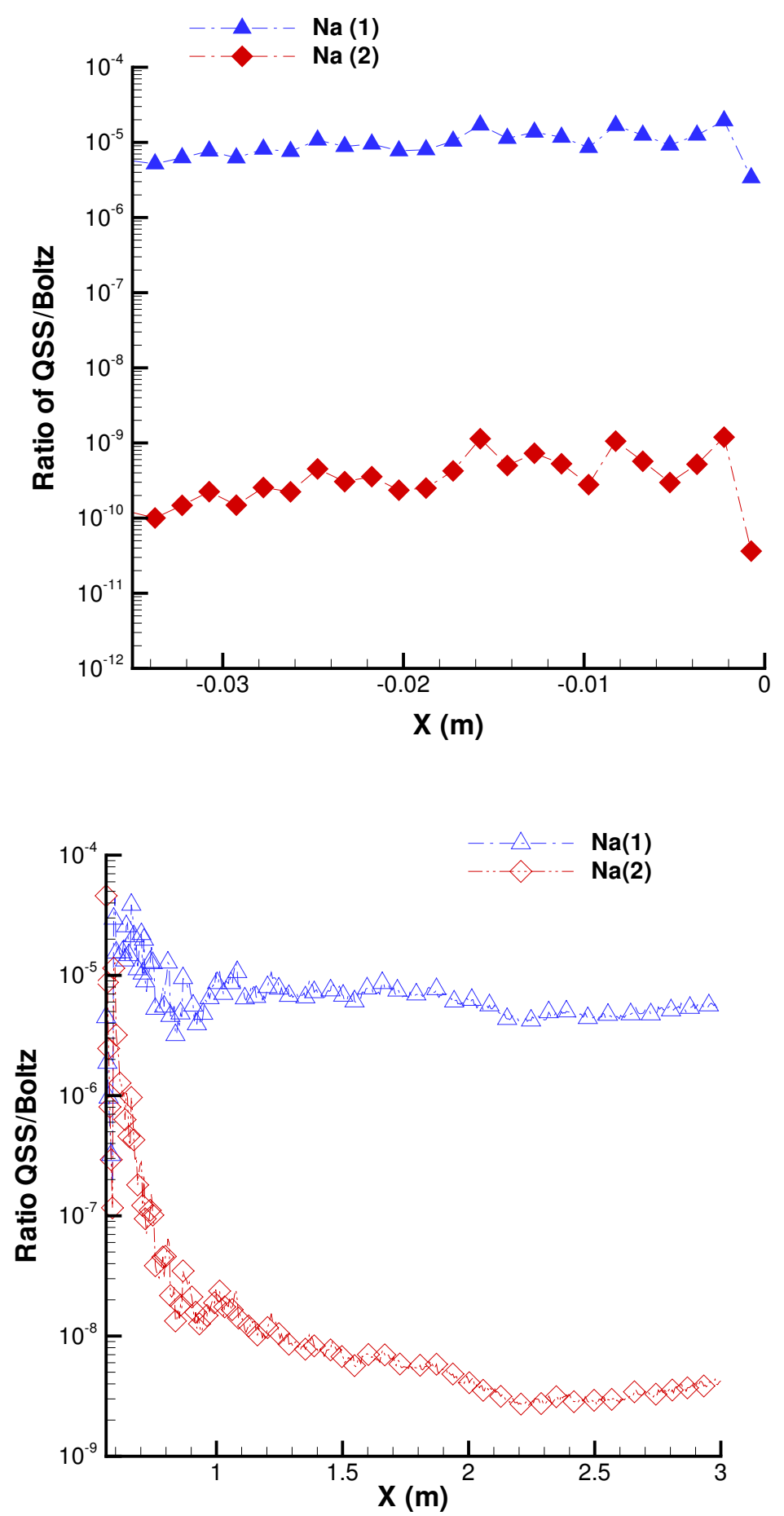

Figure 35. The ratio of QSS/Boltz of sodium excitation states at ${ }^{2} \mathbf{P}_{1 / 2}$ and ${ }^{2} \mathbf{D}_{5 / 2}$ along the forebody (top) and afterbody (bottom) stagnation line at $81 \mathrm{~km}$. 